\title{
Novel xylose transporter Cs4130 expands the sugar uptake repertoire in recombinant Saccharomyces cerevisiae strains at high xylose concentrations
}

João Gabriel Ribeiro Bueno ${ }^{1,2}$, Guilherme Borelli², Thamy Lívia Ribeiro Corrêa' ${ }^{1}$, Mateus Bernabe Fiamenghi ${ }^{2}$, Juliana José2 ${ }^{2}$ Murilo de Carvalho $0^{3,4}$, Leandro Cristante de Oliveira ${ }^{5}$, Gonçalo A. G. Pereira² and Leandro Vieira dos Santos ${ }^{1,2^{*}}$ (I)

\begin{abstract}
Background: The need to restructure the world's energy matrix based on fossil fuels and mitigate greenhouse gas emissions stimulated the development of new biobased technologies for renewable energy. One promising and cleaner alternative is the use of second-generation $(2 \mathrm{G})$ fuels, produced from lignocellulosic biomass sugars. A major challenge on $2 \mathrm{G}$ technologies establishment is the inefficient assimilation of the five-carbon sugar xylose by engineered Saccharomyces cerevisiae strains, increasing fermentation time. The uptake of xylose across the plasma membrane is a critical limiting step and the budding yeast $S$. cerevisiae is not designed with a broad transport system and regulatory mechanisms to assimilate xylose in a wide range of concentrations present in $2 \mathrm{G}$ processes.

Results: Assessing diverse microbiomes such as the digestive tract of plague insects and several decayed lignocellulosic biomasses, we isolated several yeast species capable of using xylose. Comparative fermentations selected the yeast Candida sojae as a potential source of high-affinity transporters. Comparative genomic analysis elects four potential xylose transporters whose properties were evaluated in the transporter null EBY.WW4000 strain carrying the xylose-utilizing pathway integrated into the genome. While the traditional xylose transporter Gxf1 allows an improved growth at lower concentrations (10 g/L), strains containing Cs3894 and Cs4130 show opposite responses with superior xylose uptake at higher concentrations (up to $50 \mathrm{~g} / \mathrm{L}$ ). Docking and normal mode analysis of Cs4130 and Gxf1 variants pointed out important residues related to xylose transport, identifying key differences regarding substrate translocation comparing both transporters.

Conclusions: Considering that xylose concentrations in second-generation hydrolysates can reach high values in several designed processes, Cs4130 is a promising novel candidate for xylose uptake. Here, we demonstrate a novel eukaryotic molecular transporter protein that improves growth at high xylose concentrations and can be used as a promising target towards engineering efficient pentose utilization in yeast.
\end{abstract}

\footnotetext{
*Correspondence: leandro.santos@Inbr.cnpem.br

${ }^{1}$ Brazilian Biorenewable National Laboratory (LNBR), Brazilian Center

for Research in Energy and Materials (CNPEM), Campinas, São Paulo

13083-100, Brazil

Full list of author information is available at the end of the article
}

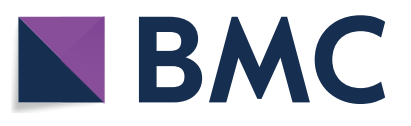

(c) The Author(s) 2020. This article is licensed under a Creative Commons Attribution 4.0 International License, which permits use, sharing, adaptation, distribution and reproduction in any medium or format, as long as you give appropriate credit to the original author(s) and the source, provide a link to the Creative Commons licence, and indicate if changes were made. The images or other third party material in this article are included in the article's Creative Commons licence, unless indicated otherwise in a credit line to the material. If material is not included in the article's Creative Commons licence and your intended use is not permitted by statutory regulation or exceeds the permitted use, you will need to obtain permission directly from the copyright holder. To view a copy of this licence, visit http://creativeco mmons.org/licenses/by/4.0/. The Creative Commons Public Domain Dedication waiver (http://creativecommons.org/publicdomain/ zero/1.0/) applies to the data made available in this article, unless otherwise stated in a credit line to the data. 
Keywords: Xylose, Xylose transporter, Major facilitator superfamily, Saccharomyces cerevisiae, Pentose metabolism, Industrial biotechnology

\section{Background}

Concerns regarding the mitigation of climate change and global warming impacts have led to a commitment to restructure the energy matrix based on fossil fuels. One promising and cleaner energy alternative demands the development of sustainable advanced biofuels produced from renewable lignocellulosic biomass, also known as second-generation (2G) biofuels [1-4]. The conversion of plant biomass sugars into bioproducts requires the extraction and breakdown of the main macromolecular components from the recalcitrant structure of the plant cell wall. The complex architecture of plant cell wall contains cellulose (40-50\%), hemicellulose $(25-35 \%)$, and lignin $(15-20 \%)$ as major constituents [5, 6]. Cellulose consists of chains of glucose, the most abundant sugar monomer in biomass, while the hemicellulosic fraction contains xylose, a fivecarbon sugar, as the main component, being the second most abundant monomer in the lignocellulosic portion.

The conversion of biomass-derived sugars into different bioproducts requires the development of robust and high-yielding microbial platforms through synthetic biology approaches. The budding yeast Saccharomyces cerevisiae has been traditionally used as a eukaryotic platform model to design new routes and engineer metabolic fluxes towards desired high-value bioproducts, such as fuels, chemicals, food, feed, and pharmaceuticals $[7,8]$. However, the C5 sugar xylose is not naturally metabolized by wild-type strains of $S$. cerevisiae, requiring systems metabolic engineering strategies to develop high-performance strains $[9,10]$. Xylose catabolism in S. cerevisiae occurs through the integration of heterologous metabolic pathways associated with adaptive evolution strategies to reshape cellular metabolism and improve fermentation fitness [10-13]. Mutations related to fitness-enhanced phenotypes arise during evolution to alleviate metabolic bottlenecks and speed up xylose conversion [10, 12]. By using these combined approaches, it is possible to improve catabolic fluxes and engineer strains to assimilate xylose more efficiently. However, several limitations still need to be addressed to optimize biomass conversion, including the inefficient transport of xylose by $S$. cerevisiae strains.

The transport of sugars is mediated through transmembrane proteins, which perform the uptake of a broad range of substrates between extracellular and intracellular environments of the cell. Sugar transporters are found mainly in the major facilitator superfamily (MFS), spread in all branches of life [1417]. The MFS members are divided into three classes: uniport, transports a single substrate; symporter, the transport of the substrate is usually coupled to an ion; and antiport, which transports two different substrates in opposite directions $[14,15,18]$. MFS transporters are branched in 74 subfamilies [19], some of them including the well-known xylose transporters GAL2 [20-24], HXT [20-23], GXF1 [21, 25, 26] and GXS1 [27-29]. The budding yeast $S$. cerevisiae has a set of native hexose transporters (Hxt1p, Hxt2p, Hxt4p, Hxt5p, Hxt7p, and Gal2p) which can also import xylose, but with low affinity and strong glucose repression, limiting fermentation of mixed biomass sugars $[30,31]$. S. cerevisiae endogenous transporters have a preference to assimilate glucose over xylose in industrial lignocellulosic hydrolysates, increasing the time of fermentation [32]. The expression of heterologous xylose transporters in engineered S. cerevisiae strains has already resulted in improved xylose assimilation rates. For example, the most well-described facilitator, Gxf1, isolated from Candida intermedia, improved transport kinetics and xylose utilization in recombinant xylose-fermenting S. cerevisiae by threefold $[25,26,33-35]$. The glucose/ xylose-facilitator Gxf1 from C. intermedia is considered the most efficient transporter when compared to other known heterologous xylose transporters, such as Sut1, Xut1, Xut3 (Scheffersomyces stipitis), At5g59250 (Arabidopsis thaliana) and XylHP (Debaryomyces hansenii) [21, 26, 33]. Interestingly, the transport of xylose in Gxf1 seems to be concentration-dependent given that improvements in transport rates and growth were observed only at very low xylose concentrations $(\sim 4 \mathrm{~g} / \mathrm{L})$, while remained unchanged at higher concentrations $[21,25,33]$.

The inefficient and unspecific xylose transport across the plasma membrane in S. cerevisiae is a major drawback to design microbial platforms for industrial biomass conversion [36]. Identifying superior transporter proteins and molecular mechanisms for improved xylose utilization through metabolic engineering is essential for the development of $2 \mathrm{G}$ biorefineries. In this work, we described a novel membrane transporter protein that improves growth in high xylose concentrations and can be used to engineer $S$. cerevisiae strains for efficient pentose conversion in biobased fuels and chemicals. 


\section{Results}

\section{Isolation and molecular identification of $\mathrm{C} 5$ yeasts}

Only a small number of heterologous sugar transporters capable of enhancing xylose uptake in $S$. cerevisiae have been identified and characterized so far. In order to explore novel yeast C5 uptake systems, we accessed the microbiome of potential sources such as decayed energy cane and sugarcane, insect plagues isolated from these materials and also from corn crops (Additional file 1: Figure S1). A total of 300 yeast colonies were recovered from enrichment in xylose medium and molecularly identified based in D1/D2 domains and the ITS region. The consecutive batches using the xylose isolation medium were employed to enrich the population and select only strains capable of assimilating xylose more efficiently, resulting in only a few species from each material that dominated the population. The presumable wide diversity in populations colonizing the starting samples was probably severely reduced in response to a selective xylose-enriched environment. Additional file 2: Table S1 summarizes the 13 yeast species identified and source of isolation, confirming the dominance of few species in each material, resulting from adaptation and competition regarding the use of xylose as the sole carbon source.

\section{Comparative xylose fermentation}

The enriched yeast culture libraries represent a potential source of novel specific transport systems for xylose. To discover novel potential gene donor candidates for heterologous expression of C5 transporters in S. cerevisiae, comparative fermentation assays using xylose as the sole carbon source were performed in order to measure fitness and xylose consumption rates. All species were able to consume xylose and produce xylitol preferably, with low concentrations of glycerol and ethanol also observed as fermentation byproducts. Xylitol was the main product observed due to a redox cofactor imbalance caused by a xylose reductase with a higher affinity to NADPH and a xylitol dehydrogenase $\mathrm{NAD}^{+}$dependent, a pattern traditionally present in wild C5 yeasts. The kinetic parameters for fermentation assays are summarized in Additional file 2: Table S1. All yeast cells were able to consume xylose with a wide difference in consumption rates. After $54 \mathrm{~h}$ of experiment, starting with $20 \mathrm{~g} / \mathrm{L}$ of xylose, the differences ranged from 28.5 to $100 \%$ of xylose consumed. The four yeasts from Candida genus presented the best performances regarding the assimilation of xylose with approximately $90 \%$ of the sugar consumed for C. boidinii and $100 \%$ for the others in the group. Except for C. boidinii, the other Candida yeasts presented the highest yields of xylitol with a maximum of $\sim 0.67 \mathrm{~g}$ xylitol/g xylose produced by C. sojae. Wickerhamomyces anomalus also performed well in terms of xylitol production, with a yield of
0.54 g xylitol/g xylose. In YPX containing $30 \mathrm{~g} / \mathrm{L}$, under aerobic conditions, the $C$. sojae isolate consumed all the sugar in approximately $70 \mathrm{~h}$, starting with a low cell density, producing xylitol as the main product followed by very low concentrations of ethanol and glycerol (Additional file 3: Figure S2). We hypothesized that, since $C$. sojae displayed a fast xylose consumption compared to the other strains, this isolate could be a promising source of novel xylose transporter-encoding genes for heterologous expression in $S$. cerevisiae.

\section{Genome mining of xylose transporter-encoding genes from C. sojae}

A set of known genes which encode microbial xylose transporters were retrieved from public databases and used as baits to screen putative promising transporters in the $C$. sojae genome and classify as symporter or facilitator. The transporter genes phylogeny indicate that all xylose transporters are homologous, i.e., have a unique ancestor gene (Fig. 1). Gene families attributed by OrthoMCL pipeline revealed as monophyletic groups in the whole gene tree, supporting that those families are homolog groups with independent evolutionary trajectories. The only exception was Aa1938, which was closer to Fam4266 than to the rest of Fam63, with whom it was clustered in OrthoMCL (Fig. 1a). Four of the five gene families had at least one known xylose transporter clustered in their clades allowing some inference on the phenotype of each family genes. Comparing the yeast transporter genes with other fungi, the mstA transporter from Aspergillus niger is in the family 5386 tree and grouped with a Lipomyces starkeyi transporter (Ls6310). This observed relationship is not expected considering the species' phylogeny as A. niger is an Ascomycete from a different Class. In the same way, the bacterial transporter glcp from Bacillus subtilis clustered with family 8's yeast transporters instead of with other bacterial transporters as would be expected. The incongruence among gene trees and the species phylogeny may arise from different factors, such as horizontal gene transfers, incomplete lineage sorting, and also similar selective pressures leading to sequence convergence.

Gene family expansions are easily recognized throughout the yeast transporter's phylogeny (Fig. 1). In family 8, Cs3894 and Cs4130 appear as copies from a putative gene duplication in the base of Pichia, Spathaspora and Candida clade (Fig. 1b), where the first shares a subclade that follows the species' phylogeny [37] and the second in a subclade exclusive of C. sojae, C. tropicalis, and C. tanzawanensis, putatively being lost in Pichia and Spathaspora lineages. In family 4266, Cs186 is a putative result of many duplication events inside the $C$. sojae lineage (Fig. 1d). The high duplication rate coupled with high 


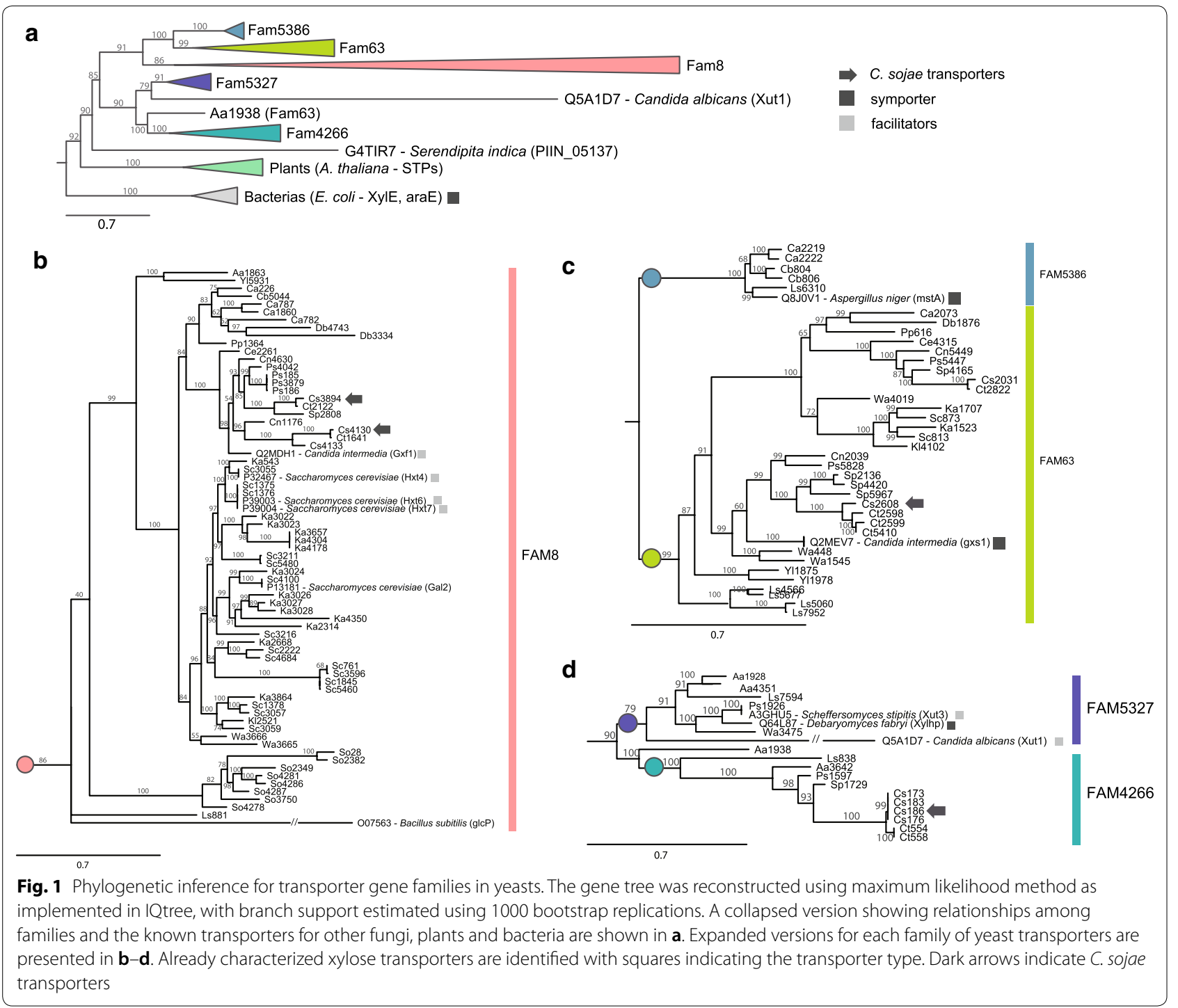

conservation of these genes suggests that these transporters are dosage-dependent as reported for hexose transporters [38]. For dose-dependent genes, it is potentially adaptive to increase gene number maintaining sequences features because it implies in higher transcription and a larger number of proteins working in the cell. In this way, transporter genes that undergo recent duplication events might be evolving under a selective process. By using an evolutionary approach for prospecting xylose transporters candidates for industrial applications, we hypothesize that transporters from our dataset more closely related to xylose transporters known from literature would have a similar capacity on uptaking this pentose. We then selected closely grouped genes to these transporters as candidates, such as Cs4130 and Cs3894 (close to GXF1), as well as some more distantly grouped-Cs186, a whole orthogroup distant from XUT1, XUT3 and
XylhpI; and Cs2608, close to GXS1. Based on sequence, Cs186, Cs3894 and Cs4130 are facilitators and Cs2608 is a symporter.

\section{C. sojae transporters mediate uptake of different carbon sources}

The substrate spectrum from the selected sugar transporters Cs186, Cs2608, Cs3894, and Cs4130 was evaluated in the strain EBY_Xyl1, a modified yeast cell derived from EBY.VW4000, which lacks transporters of the families HXT1-17 and GAL2, and is unable to grow on glucose as the sole carbon source [39]. EBY_Xyl1 was constructed with the integration of an expression cassette containing the genes XYL1 and XYL2 from S. stipitis and an additional copy of xylulokinase (XKS1). The three genes were expressed under the control of different strong constitutive promoters of genes from the glycolytic pathway of 
S. cerevisiae (Additional file 4: Table S2). The candidate transporters were cloned in a multi-copy vector pRS426 using the promoter and terminator regions of TDH1 from $S$. cerevisiae. As a positive transport-system control, the traditional xylose-facilitator Gxf1 from C. intermedia was chosen, since this transporter is well-known as the best heterologous xylose transporter already described in the literature [21, 33]. The plasmids expressing the cloned putative $\mathrm{C} 5$ transporters were used to transform the EBY_Xyl1 yeast cell. The candidate transporters were fused to a codon-optimized GFP-mUkG1 sequence to confirm the correct cellular localization in the yeast cell membrane and observed by confocal microscopy. Yeast cells expressing the GFP-tagged plasmids presented a fluorescence halo in the cell periphery indicating the correct localization of the transporters in the cell membrane (Fig. 2). However, the presence of fluorescence in the cytoplasm in a few yeast cells was observed, indicating possible degradation through a regulatory post-translational modification [40] or an unfolded protein response (UPR) triggered by overexpression of the heterologous transporter protein [41].

We analyzed the substrate spectrum of EBY_Xyl1 mutants carrying the specified sugar transporters using six different carbon sources on solid culture medium - $2 \%$ maltose (control), mannose, fructose, xylose, glucose, and galactose. The transformants were grown overnight to the exponential phase and spotted in tenfold serial dilutions onto solid culture medium. This analysis showed that $C$. sojae transporters restored the growth of EBY_Xyl1 strain in all sugars evaluated (Fig. 3). Curiously, the control strain EBY_Xyl1 carrying pRS426 plasmid showed a slight background on galactose and xylose. Parental strain EBY.VW4000 was previously described to show basal growth on galactose [39]. As this background was similar comparing these two sugars, the remaining transporters on the EBY.VW4000 strain which confers growth on galactose probably could be responsible for this behavior observed for xylose. However, for the other sugars, except maltose (control), no growth was demonstrated using the control EBY_Xyl1_pRS426. All transporters were functional and able to restore growth in all evaluated carbon sources showing a broad substrate affinity. The only exception was Cs186, which presented a slight background growth similar to pRS426 control on xylose, indicating that this transporter is unable to assimilate this sugar. Cs3894 and Gxf1 were able to support better and similar patterns of growth on glucose, mannose and fructose compared to the other transporters. The strain expressing the Cs2608 presented a poor growth on mannose and glucose compared to the other substrates. Cs3894 and Cs4130 presented a superior growth on $20 \mathrm{~g} / \mathrm{L}$ of xylose and, interestingly and not expected, the growth of the strain expressing Cs4130 was better on xylose than glucose.
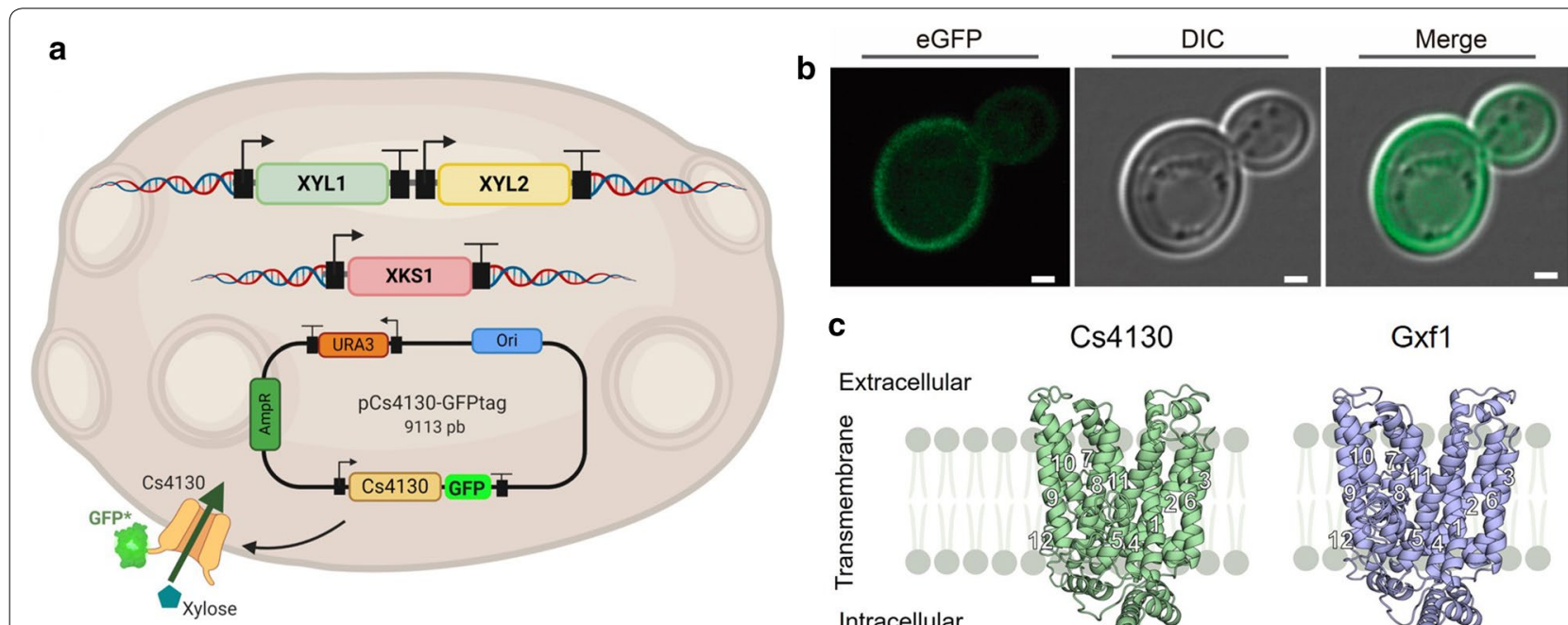

C

Cs4130

Gxf1
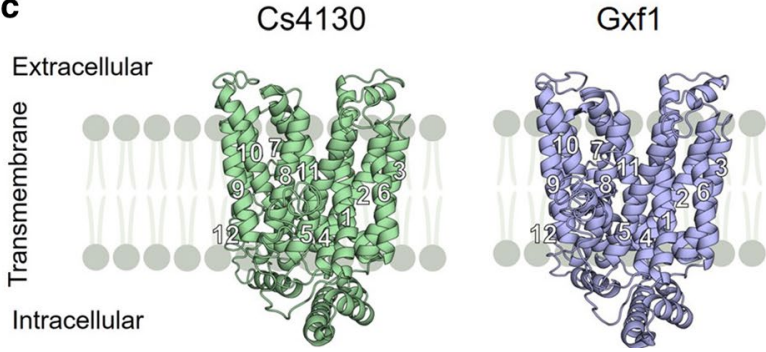

Fig. 2 Subcellular localization of tested transporters in S. cerevisiae cells. a Design of EBY_Xyl1 cells with an integrated XR/XDH/XKS pathway and a multi-copy vector expressing the transporter fused with a codon-optimized eGFP-ymUkG1 (Created with BioRender). b A confocal image confirms the presence of a fluorescence halo in the cell periphery indicating the correct localization of the transporter in the cell membrane. DIC and fluorescence images were taken by a confocal microscope and merged. Scale bars $=1 \mu$ m. c Crystallographic and modeled structures of Cs4130 and Gxf1, respectively, embedded in the cytoplasmatic membrane. Cs4130 and Gxf1 show the typical fold of MFS transporters. The 12 transmembrane a-helix in Cs4130 and Gxf1 were assigned based in XyIE 


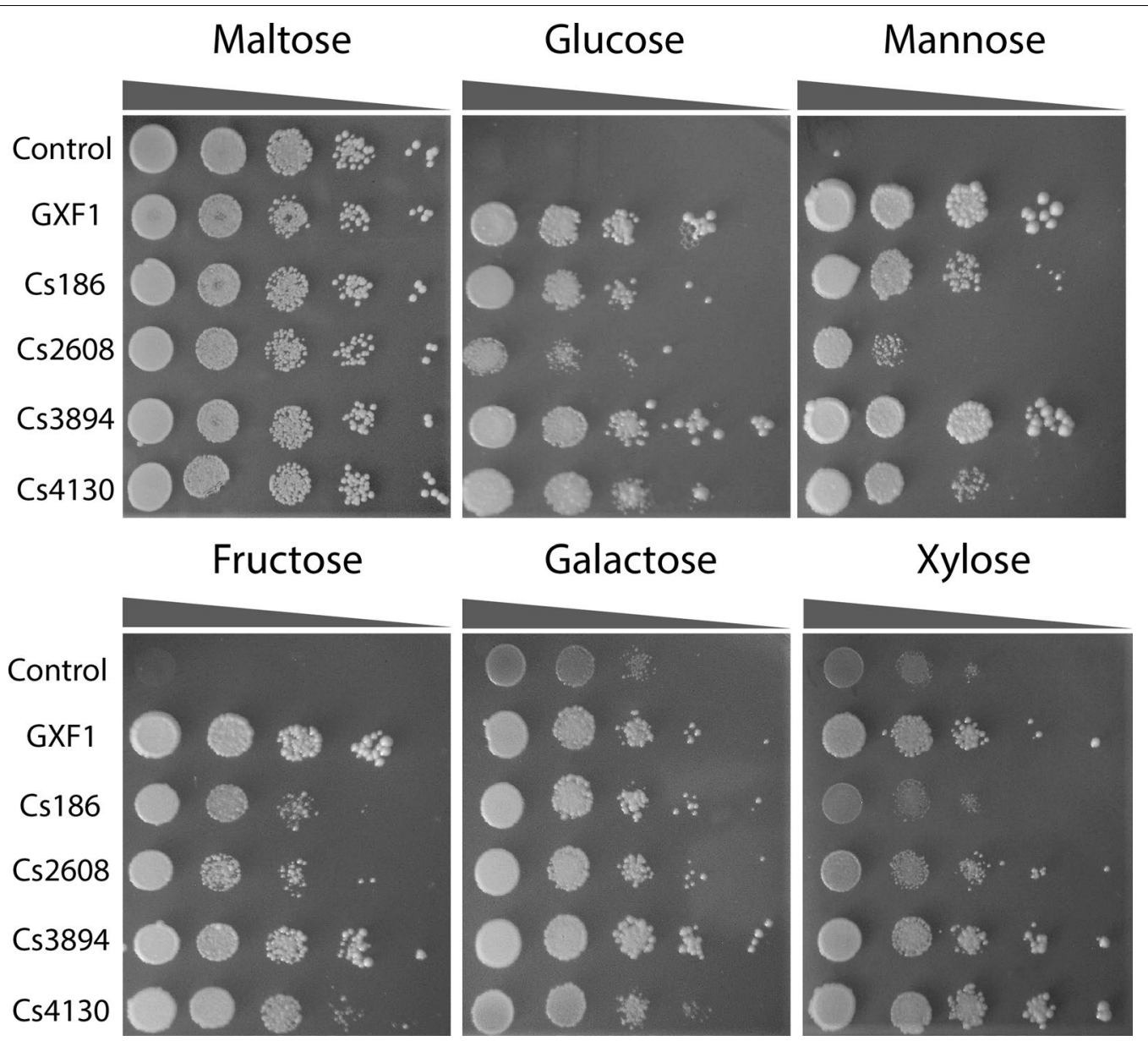

Fig. 3 Functional profile of sugar affinities of C. sojae transporters. Drop-assay analysis on different sugars, $20 \mathrm{~g} / \mathrm{L}$, of EBY_Xyl1 strain expressing the indicated transporters and the control vector pRS426. The cultures were incubated at $30^{\circ} \mathrm{C}$ for 3 days (maltose), 5 days (glucose, mannose, fructose and galactose) or 7 days (xylose). Yeast cells inocula were grown in YNB complete medium without uracil supplemented with maltose for $24 \mathrm{~h}$. Initial $\mathrm{OD}_{600}$ was established at 1 before a serial dilution. All experiments were performed in triplicate

\section{Growth at different ranges of xylose concentrations}

The growth of EBY_Xyl1 mutants carrying C. sojae transporter genes and GXF1 as positive control were compared in solid medium with $0.5,1,2,3$ and $5 \%$ of xylose as the sole carbon source (Fig. 4a). The facilitator Gxf1 restored growth in all xylose concentrations evaluated. Analyzing the results of $\mathrm{Gxf1}$, it is possible to notice a decrease in growth with increasing concentrations of xylose. Although this facilitator allows a higher growth of EBY_Xyl1, compared to all other C. sojae transporters on low xylose concentrations, 5 and $10 \mathrm{~g} / \mathrm{L}$, on intermediary and high concentrations, inhibition was observed. Surprisingly, the C. sojae transporters Cs3894 and Cs4130 demonstrated an opposite behavior allowing improvements on growth in intermediary and higher xylose concentrations, $30 \mathrm{~g} / \mathrm{L}$ and $50 \mathrm{~g} / \mathrm{L}$, respectively (Fig. 4a). The EBY_Xyl1 cell expressing Cs4130 showed a better growth pattern compared to the other transporters in high concentrations of xylose, showing a natural loss of inhibition in contrast to commonly observed in Gxf1. The spot-assay experiment in different xylose concentrations were repeated three times, and each experiment was conducted in triplicate to confirm the growth pattern.

The spot-assay results were also confirmed in liquid media in a high-cell density fermentation assay using EBY_Xyl1_pRS426 as a control strain and xylose as the only source of carbon. Since Cs4130 presented the most interesting results, we focused further experiments in this facilitator. Yeast cells expressing Cs4130 were able to assimilate xylose and grow in both 10 and $50 \mathrm{~g} / \mathrm{L}$, although a higher xylose consumption rate was observed in superior concentrations (Fig. 4b, c). For both concentrations, in the first hours of analysis, productivity was higher, but decreased during fermentation, probably due to redox balance limitations from the $\mathrm{XR}-\mathrm{XDH}$ pathway. As expected, cells expressing Gxf1 maintained the same 
a

\section{Xylose}

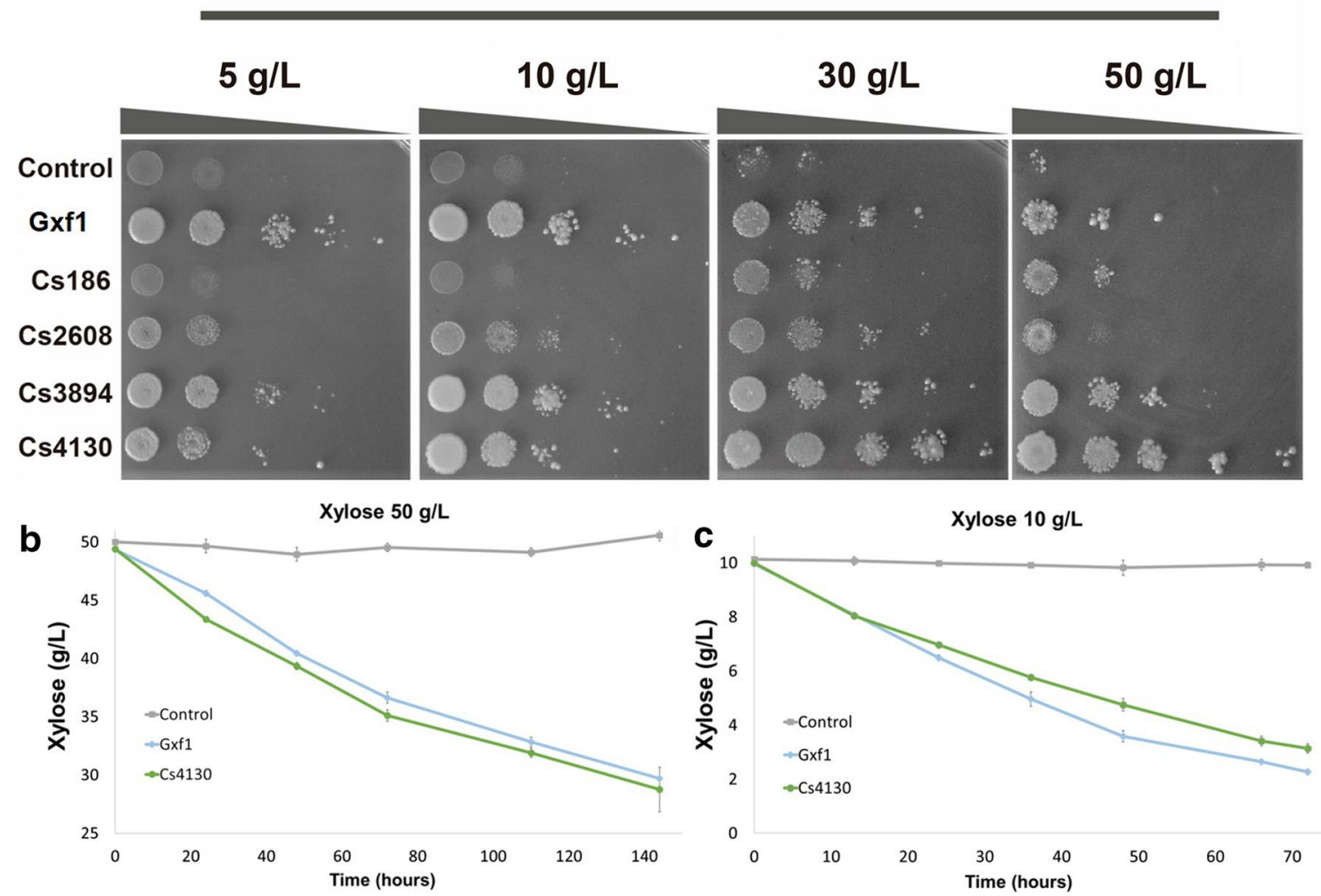

Fig. 4 Functional profile of concentration-dependence of C. sojae transporters. a Growth on different range of xylose concentrations showing an uptake/concentration dependency. All experiments were performed in triplicate. High-cell density fermentation of Cs4130 and Gxf1 in xylose at $50 \mathrm{~g} / \mathrm{L}$ (b) and $10 \mathrm{~g} / \mathrm{L}$ (c). The fermentations were carried out on YNBX medium at $30^{\circ} \mathrm{C}, 200 \mathrm{rpm}$. Control represents the EBY_Xyl1 harboring empty vector pRS426. Error bars represent standard deviation from average measures from triplicates

consumption profile observed in the spot-assay. Comparing both transporters on $50 \mathrm{~g} / \mathrm{L}, \mathrm{Cs} 4130$ allowed faster assimilation with an increase of $30 \%$ in xylose consumption rate compared to Gxf1 in $24 \mathrm{~h}$. However, on lower xylose concentrations, Gxf1 allowed a faster growth and improved xylose consumption rate by $49,5 \%$ in $24 \mathrm{~h}$ (Fig. 4b, c).

\section{Simultaneous consumption of glucose and xylose on Cs4130}

Simultaneous consumption of glucose and xylose in a high-cell density fermentation using a C6/C5 mixture was also evaluated, with $10 \mathrm{~g} / \mathrm{L}$ of each sugar and EBY Xyl1 strain expressing Cs4130 and Gxf1 (Fig. 5a). This fermentation showed a faster glucose consumption in the first $10 \mathrm{~h}$. A very small amount of xylose was consumed in this period, confirming, as expected, a higher affinity for glucose compared to xylose. As an example, the facilitator Gxf1 is known to have a higher glucose affinity $\left(K_{\mathrm{m} \text { glu- }}\right.$ cose $=2.0 \pm 0.6)$ compared to xylose $\left(K_{\mathrm{m} \text { xylose }}=48.7 \pm 6.5\right)$, [34]. The preferential glucose consumption confirmed that Cs4130 does not bypass glucose inhibition, similarly as observed for Gxf1. As the concentrations used were low $(10 \mathrm{~g} / \mathrm{L})$, cells expressing Gxf1 consumed the sugars faster.

To further evaluate the glucose inhibition of Cs4130, from EBY_Xyl1, the strain EBY_Xyl1_hx $k^{0}$ was constructed by disrupting the glycolytic pathway through gene knockout of three hexokinases: HXK1, HXK2 and GLK1, and further restored sugar uptake by expression of individual heterologous transporters, Cs4130 and GXF1. The derived strain assimilated glucose from extracellular media but was not capable to metabolize this sugar as a carbon source (Fig. 5). Therefore, it is a platform to analyze glucose inhibition in a sugar mixture. Since glucose or maltose cannot be used for growth, we used 3\% ethanol as a positive control. EBY_ Xyl1_hx $k^{0}$ strains harboring individual heterologous transporters, showed, as expected, growth on xylose and impairment on glucose uptake. The glucose inhibition test was performed in three different concentrations of C5/C6 mixtures on solid media. The xylose 

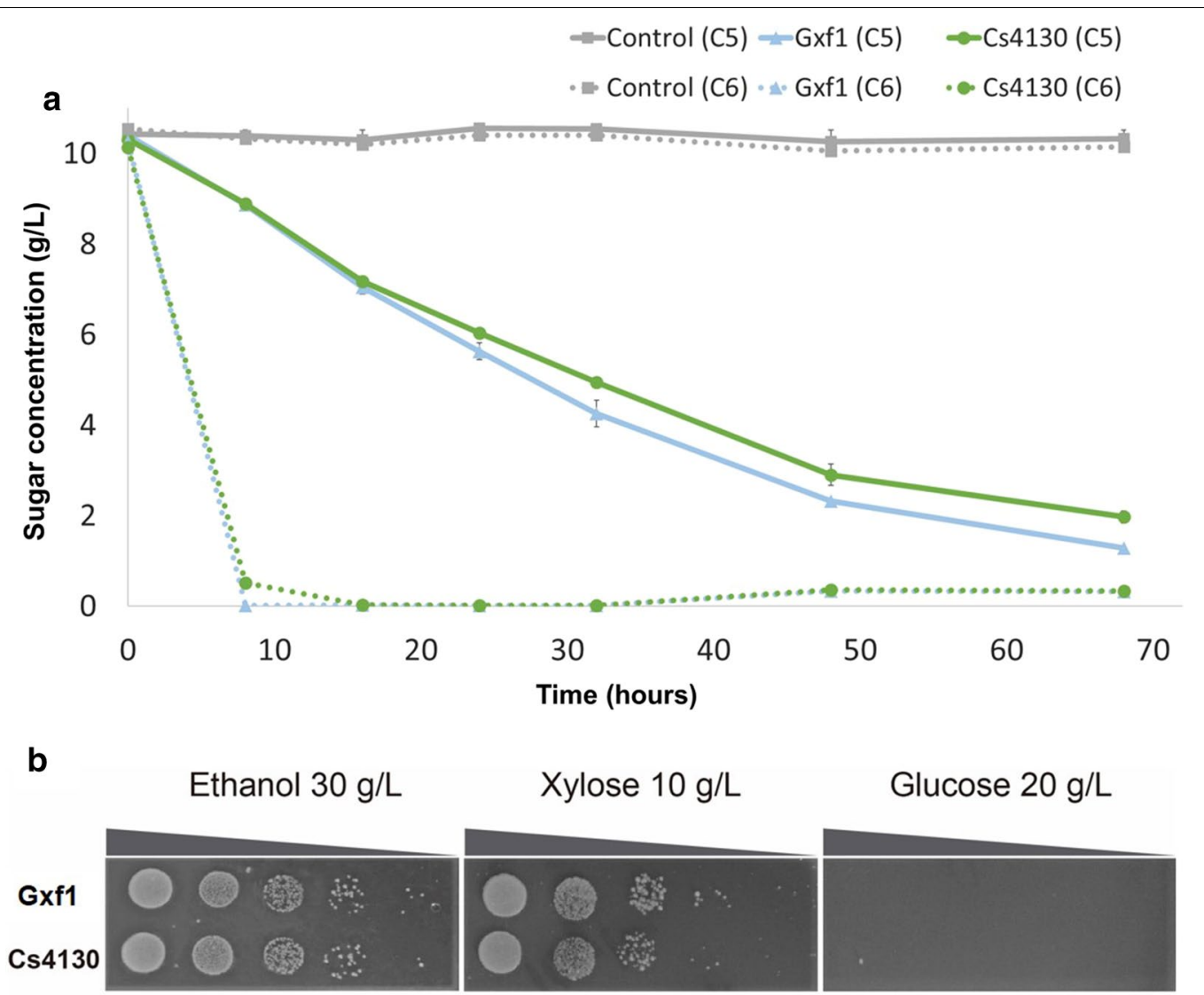

Xylose $10 \mathrm{~g} / \mathrm{L}$

Glucose $20 \mathrm{~g} / \mathrm{L}$
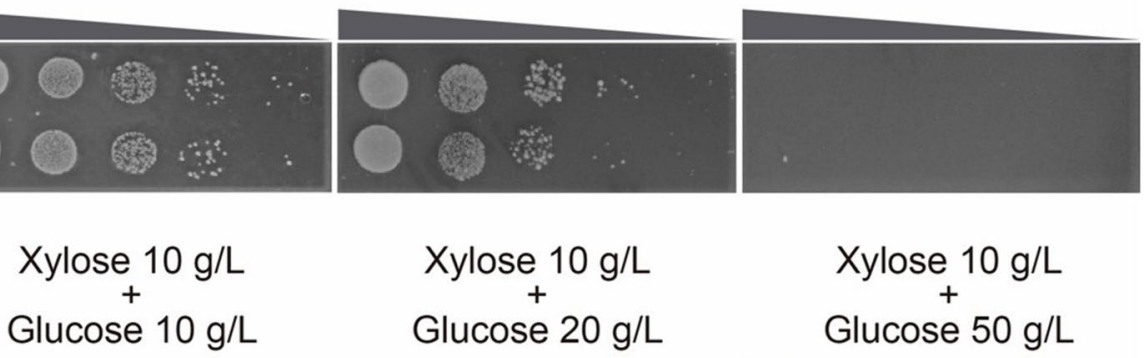

Xylose $10 \mathrm{~g} / \mathrm{L}$ Glucose $20 \mathrm{~g} / \mathrm{L}$

Xylose $10 \mathrm{~g} / \mathrm{L}$

Glucose $50 \mathrm{~g} / \mathrm{L}$

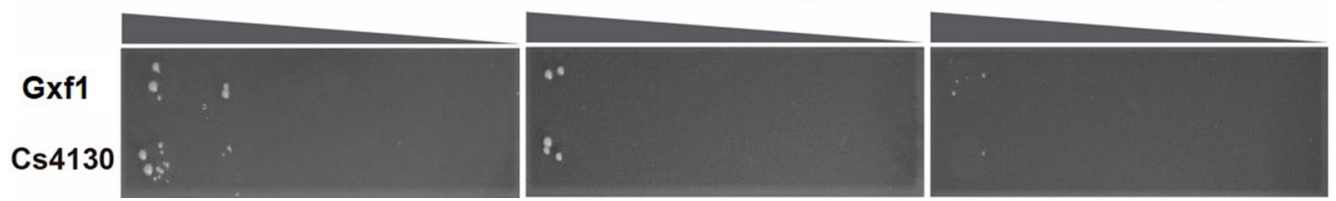

Fig. 5 Glucose inhibition of Cs4130 in glucose and xylose mixtures. a High-cell density fermentation of Cs4130 and Gxf1 in xylose/glucose co-fermentation. Xylose and glucose consumption are represented by full and dotted lines, respectively. Fermentations were conducted on complete medium with $10 \mathrm{~g} / \mathrm{L}$ of xylose and $10 \mathrm{~g} / \mathrm{L}$ of glucose at $30^{\circ} \mathrm{C}, 200 \mathrm{rpm}$. Control represents the EBY_Xyl 1 harboring empty vector pRS426. Error bars represent standard deviation from average measures from triplicates. $\mathbf{b}$ Drop-assay analysis of EBY_Xyl1_hxk $k^{0}$ strain expressing the indicated transporters. Cells were grown on complete medium without uracil supplemented with $3 \%$ ethanol for $56 \mathrm{~h}$ before a serial dilution. The initial $\mathrm{OD}_{600}$ was established on 1. Plates were incubated at $30^{\circ} \mathrm{C}$ for 10 days

concentration was maintained on $10 \mathrm{~g} / \mathrm{L}$ and glucose was added to final concentrations of $10 \mathrm{~g} / \mathrm{L}, 20 \mathrm{~g} / \mathrm{L}$ and $50 \mathrm{~g} / \mathrm{L}$, respectively. The inhibition test showed that yeast cells expressing both transporters were unable to restore significant growth on the different mixtures, even on low glucose concentrations (Fig. 5b). This phenotype indicates that these heterologous transporters show a natural higher preference for glucose over xylose, which leads to growth impairments on $h x k^{0}$ strains on the presence of both sugars.
Docking and normal mode analysis (NMA) point out different behavior of xylose-bound Cs4130 and Gxf1

The modeled structures of Cs4130 and Gxf1 were acquired in the I-TASSER server [42], both showing the typical fold of MFS transporters: 12 transmembrane (TM) $\alpha$-helix with the N- (TM1-TM6) and C- (TM7-12) termini facing the intracellular side [14] (Fig. 2c). High TM-scores $(>0.6)$ were recorded for GLUT transporters, typical of mammals (PDB codes: 5C65, 4YBQ and 4PYP); XylE and $\mathrm{GlcP}_{\mathrm{SE}}$, from bacteria (4GBY, 4LDS); 
and STP10, from plant (6H7D) for both Cs4130 and Gxf1 models. XylE, a D-xylose: $\mathrm{H}^{+}$symporter found in Escherichia coli shows 27.02 and $25.56 \%$ identity (amino acids) with Cs4130 and Gxf1, respectively. XylE was adopted as a facilitator model throughout this work given the availability of its outward-facing/partly occluded crystallographic structure bound to xylose (4GBY) as well as the distinction of some residues essential for sugar transport [43], which was extended to Cs4130 and Gxf1 in this work.

The docking analysis of Cs4130 and Gxf1 with glucose, fructose, galactose, mannose and xylose showed that all these sugars can bind to both transporters (Table 1). Cs4130, Gxf1 and XylE show higher binding affinities (lower binding energies $[\mathrm{kcal} / \mathrm{mol}]$ ) to glucose than xylose. Regarding xylose, a slightly low binding energy was recorded for Cs4130 compared to Gxf1 and XylE (Table 1). Mutation of specific residues in XylE leads to a decrease or abrogation of xylose transport [43]. Their counterparts in Cs4130, Gxf1, STP10 (from A. thaliana) and $\mathrm{GlcP}_{\mathrm{SE}}$ (from Staphylococcus epidermidis) are strongly conserved suggesting their importance for sugar transport (Fig. 6, Additional file 5: Table S3).

An extensive comparison of XylE with Cs4130 and Gxf1 was made to infer the molecular mechanisms related to the improved xylose assimilation by Cs4130 over Gxf1 at higher concentrations. The distance to xylose (DtX, $\AA$ ) of all residues mutated in XylE [43] was measured using $4 \mathrm{GBY}$ as reference (Table 2) to understand how the residues interact with xylose. Given the conservation/superposition of the residues mutated in XylE in both Cs4130 and Gxf1 (Fig. 7, Additional file 5: Table S3), the distance to the ligand was considered as the same for all targets (Table 2). Thus, the xylose binding was evaluated by docking (residues close to xylose) or normal mode analysis (residues far from xylose). It is noteworthy that small variations in the binding energy (around $0.6 \mathrm{kcal} / \mathrm{mol}$ ) of wild-type (WT) XylE compared to the variants Q168A, Q288A, N289A, N294A, Y298A, W392A, Q415A and W416A (near to xylose, $<5 \AA$ ) may be enough to abrogate

\section{Table 1 Binding energies (kcal/mol) of Cs4130, Gxf1 and XylE with monosaccharides through molecular docking analysis}

\section{GRID SCORE (kcal/mol)}

Transporter monosaccharides

\begin{tabular}{llllll}
\hline & Glucose & Fructose & Galactose & Mannose & Xylose \\
\hline Cs4130 & -32.9 & -29.7 & -30.5 & -31.6 & -28.8 \\
GXf1 & -29.3 & -29.9 & -29.3 & -29.8 & -26.0 \\
XylE & -31.0 & -30.4 & -33.3 & -33.9 & -27.4 \\
\hline
\end{tabular}

the xylose transport as seen in Sun et al. [43]. This conclusion is corroborated by the molecular docking analysis of $\mathrm{N} 325 \mathrm{~A}$. The binding energy of the N325A is equal to the WT $(-27.4 \mathrm{kcal} / \mathrm{mol})$ supporting the molecular docking procedure by agreeing with the functional studies [43] (Table 2). When Q188 (Q168 in XylE), Q314 (Q288 in XylE), Q315 (Q289 in XylE), N320 (Y298 in XylE), Y324 (Y298 in XylE) and Y428/Y431 (W392 in XylE), N451/ N454 (Q415 in XylE) and W452/W455 (W416 in XylE) in Cs4130 and Gxf1 were mutated to alanine and evaluated through molecular docking analysis, the Cs4130 mutants Q188A (variation in the binding energy compared to WT Cs4130: $2.4 \mathrm{kcal} / \mathrm{mol})$, Q314A $(0.7 \mathrm{kcal} / \mathrm{mol})$, N320A $(0.6 \mathrm{kcal} / \mathrm{mol}), \mathrm{Y} 324 \mathrm{~A}(0.7 \mathrm{kcal} / \mathrm{mol})$ and $\mathrm{Y} 428 \mathrm{~A}$ $(0.9 \mathrm{kcal} / \mathrm{mol})$ and N451A $(0.8 \mathrm{kcal} / \mathrm{mol})$ would probably result in altered xylose transport compared to WT Cs4130 since variations in the binding energy of around $0.6 \mathrm{kcal} / \mathrm{mol}$ is sufficient to abrogate xylose transport in XylE. For Gxf1, mutants Q314A (variation in the binding energy compared to WT Gxf1: $1.1 \mathrm{kcal} / \mathrm{mol}), \mathrm{N} 320 \mathrm{~A}$ $(0.8 \mathrm{kcal} / \mathrm{mol}), Y 431 \mathrm{~A}(0.7 \mathrm{kcal} / \mathrm{mol})$, N454A $(0.6 \mathrm{kcal} /$ $\mathrm{mol})$ and W455A $(0.6 \mathrm{kcal} / \mathrm{mol})$ would have the xylose transport affected (Table 2). At this stage, it is not possible to predict if the mutations could benefit or not the xylose transport in both Cs4130 and Gxf1.

Other residues, beyond the ones mutated in XylE, were chosen in Cs4130 and Gxf1 based in their closeness to xylose $(<5 \AA)$ to be evaluated by docking analysis. Changes in xylose transport are expected in mutants I191A (variation in the binding energy compared to WT Cs4130: $1.4 \mathrm{kcal} / \mathrm{mol})$, I195A (1.9 kcal $/ \mathrm{mol})$ and F419A $(1.5 \mathrm{kcal} / \mathrm{mol})$ for Cs4130, but only in mutant I195A $(1.2 \mathrm{kcal} / \mathrm{mol})$ and $Y 431 \mathrm{~A}(0.7 \mathrm{kcal} / \mathrm{mol})$ regarding $\mathrm{Gxf} 1$ (Additional file 6: Table S4). The differences in the binding energy of variants of Cs4130/Gxf1 compared to their respective WTs (Table 2 and Additional file 6: Table S4) suggests that altered xylose transport is more likely to happen in Cs4130 than in Gxf1.

The dynamical regime of the WTs Cs4130/Gxf1 and variants (mutations in residues far from xylose) were evaluated using the non-linear Normal Mode Analysis (NOLB software) [44] through the root-mean square fluctuation (RMSF). The RMSF was calculated as the average of the 10 lowest vibrational modes and an average of all 20 frames per mode. The WTs Cs4130 and Gxf1, despite the structural similarity ( $R M S D=1.14 \AA$ ), show a different fluctuation pattern mainly in the $\mathrm{N}$ and C-terminal ends and residues $31,41-49,67,71,82-89$, 145,146, 208-216, 253-258, 281-290, 335, 394-412, 438, 459-493 and 509-515 (Fig. 7a), making the comparison between mutants of transporters not so informative as by molecular docking analysis. Consequently, the influence of residue mutations must be compared only with its own 


\section{a \\ nochara \\ Cs4130 MSVSD--ST DANPNLEDKY--ENNADPNIDAAAVDLNANQPWHAYIGIITMCVLIAFGGY 56 GXf1 MSQDSHSSGAAT PVNGSILEKEKEDSPVLQ----VDAPQKGEKDYIVISIFCFMVAFGGF 56 STP10 --_-_---MAGGAFVSEGGGG------GRSYEGGVTA--FVIMTCIVAAMGGL 37 XYlE - - MNTQYNSS--YIFSITLVATLGGL 22 Gice \\ $\alpha 1$ \\ $\alpha 2$

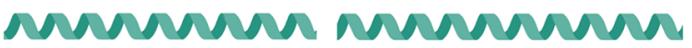 \\ CS4130 VFGFDTGTI PGFVNMTDEQRRFGS-------RRDDGTYYFSNARTGMMIGLFNAGCAV 107 GXf1 VFGFDTGTISGFVNMSDFKDRFGQ----_---HHADGTPYLSDVRVGLMISIFNVGCAV 107 \\ STP10 LFGYDLGISGGVTSMEEFLTKFFPQVESQMKKAKHDTAYCKFDNQMLQLETSSLYLAALV 97 \\ XYlE LFGYDTAVISGTV---ESLNTVFVAPQNLSE--------SAANSLLGFCVASALIGCII 70 GICP LYGYDNGVISGAL---LEIHKDIP----------------LNSTTEGIVVSSMLIGAIV 57} $::^{\star}:{ }^{\star} \cdot$

nanacion

CS4130 GALLLSKLADLYGRRFAISVSMLIYISGVIVOIA

GXf1 GGIFLCKVADVWGRRIGLMFSMAVYVVGGIIIOIS

STP10 ASFMASVITRKHGRKVSMFIGGLAFLIGALFNAF--

XYlE GGALGGYCSNRFGRRDSLKIAAVLFEISGVGSAWPELGETSINPDNTVPVYLAGYVPEFV 130

GICP GAGSSGPLADKLGRRRLVMLIAIVFIIGALILA-.. $\quad$ : ${ }^{\star \star}: \quad: \quad . \quad . \quad$ :

$\alpha 5$

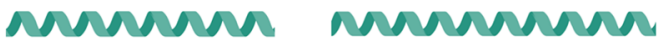

CS4130 IGRIITGVAVGTLSVVCPLFISEVSPKHLRGTLVVCFOLMITLGIFLGYCTTYGTKV--- 207

GXf1 IGRLIAGLAVGTVSVVSPLFISEVSPKQIRGTLVCCFQLCITLGIFLGYCTTYGTKT --- 207

STP10 IGRLLLGVGVGFANQSTPVYLSEMAPAKIRGALNIGFQMAITIGI LVANLINYGTSK--- 196

XYIE IYRIIGGIGVGLASMLSPMYIAELAPAHIRGKLVSFNQFAI IFGQ LLVYCVNYF IARSGD 190

GICP IGRLIIGLAVGGSMSTVPVYLSEMAPTEYRGSLGSLNQLMITIGI LAAYLVNYAF----- 154 GlCP **:: $*: . * *$

\section{$\alpha 6$}

\section{$i \alpha$}

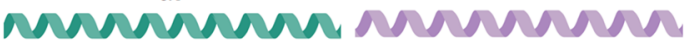

CS4130 --YSDSRQWRVPLGLCFAWALFLIGGMTRMPES PRYLLSKDKIDDARIALAKVNKASPED 265

GXf1 --YTDSRQWRIPLGLCFAWA I LLVVGMLNMPES PRYLVEKHRIDEAKRSIARSNKIPEED 265 STP10 ---MAQHGWRVS LGLAAVPAVVMV IGS F ILPDT PNSMLERGKNEEAKQMLKKIRGADNVD 253 XYIE ASWLNTDGWRYMFASECI PALLFLMLLYTVPES PRWLMSRGKQEQAEGILRKIMGNTLAT 250 GICP ---ADIEGWRWMLGLAVVPSVILLVGIYFMPES PRWLLENRNEEAAROVMKITYDDSEID 211 ia

$\alpha 7$

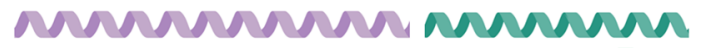

CS4130 PALLAELQLLQAGVERERLAGSATWMTLIKGKPRIFERVLVGAMLQSLQQLTGDNYFEYY 325 GXf1 PFVYTEVQLIQAGIEREALAGQASWKELITGKPKIFRRV IMGIMLQSLQQLTGDNYFFYY 325 STP10 HEFQDLIDAVEAAKK-----VENPWKNIME--SKYRPALIFCSAI PFFQQITGINVIMFY 306 XYlE OAVQEIKHSLDHG-------RKTGGR-LL--MFGVGVIVIGVMLSIFQQFVGINVVLYY 299 $\begin{array}{lll}\text { XYlE } & \text { QAVQEIKHSLDHG--------RKTGGR-LL--MFGVGVIVIGVMLSIFQQFVGINVLYY } 299 \\ \text { GICP } & \text { KELKEMKEINAIS--------ESTWTVIKS--PWLGRILIVGCIFAIFQQFIGINAVIFY } 261\end{array}$ (
$\alpha 7$

$\alpha 8$

$\alpha 9$

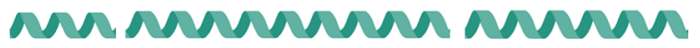

CS4130 ATMVFKAIGLDD--SFETAII IGVINFASTFVGIFAIEKLGRRLCLMLGSIGMSACFLMY 383 GXf1 GTTIFQAVGLKD--SFQTSIILGIVNFASTFVGIYVIERLGRRLCLLTGSAAMFICFIIY 383 STP10 APVLFKTLGFGDDAALMSAVITGVVNMLSTFVS IYAVDRYGRRLLFLEGGIOMFICOLLV 366 XYlE APEVEKTLGASTDIALLQTIIVGVINLTETVLAIMTVDKFGRKPLQIIGALGMAIGMFSL 359 GICP SSSIFAKAGLGEAAS ILGSVGIGT INVLVT IVA I FVVDKIDRKKLLVGGNIGMI ASLLIM 321

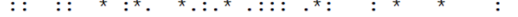

$\alpha 9$ $\alpha 10$

on

nonchora ${ }^{\alpha 11}$

CS4130 SLIGSOHLYYHGSSGET---RHRDGRAMIFVTS IYIFCFASTWAGGVYSVISELYPLKIK 440 GXF1 SLIGTQHLYKOGYSNETSNTYKASGNAMIFITCLYIFFFASTWAGGVYCIISESYPLRTR 443 STP10 GSF---IGARFGTSGTGTLTPATADWI-LAFICVYVAGFAWSWGP LGWLVPSEICPLEIR 422 XYlE GTA---FYT-------QA---PGIVALLSMLFYVAAFAMSWGPVCWVLLSEIFPNAIR 404 GlCP AIL---IWT---------IGIASSAWI I IVCLSLFIVFFGISWGPVLWVMLPELFPMRAR 369

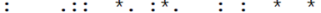

$\alpha 11$

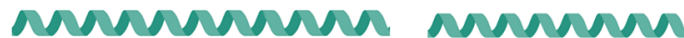

CS4130 SKAMGVANACNWLWGFLISFFTSFITD------AIHFYYGFIFMGCL-FFS IFFVYFMVY 493 GXf1 SKAMSIATAANWLWGFLISFETPFITS------AIHFYYGFVFTGCL-AFSFFYVYFFVY 496 STP10 PAGQAINVSVNMFFTELIGQFFLTMLC------HMKFG-LFYFFASMVAIMTVEIYFLLP 475 XYlE GKALAIAVAAQWLANYFVSWTFPMMDKNSWLVAHFHNGFSYWIYGCMGVLAALFMWKFVP 464 GAATGISALVLNIGTLIVSLFFPILSD------ALSTEWVELIFAFIGVLAMIFVIKFLP 423

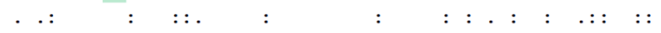

\section{$\alpha 12$}

\section{incina}

CS4130 ETKGLDLGAVEELYNAGI PAWKSAGWVPPTAQEMATTSGYARDAKPEEQQNEEQV 548 GXf1 ETKGLSLEEVDEMYASGVLPLKSASWVPPNLEHMAHSAGYAGADKATDEQV---- 547 STP10 ETKGVPIEEMGRVWKQH---WFWKKYI PEDAIIGGHD------DNNTN----- 514 XYlE ETKGKTLEELEALWEPE---TKKTQQT---ATL------------------ 491

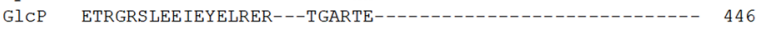

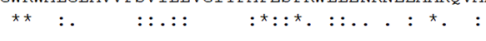

b

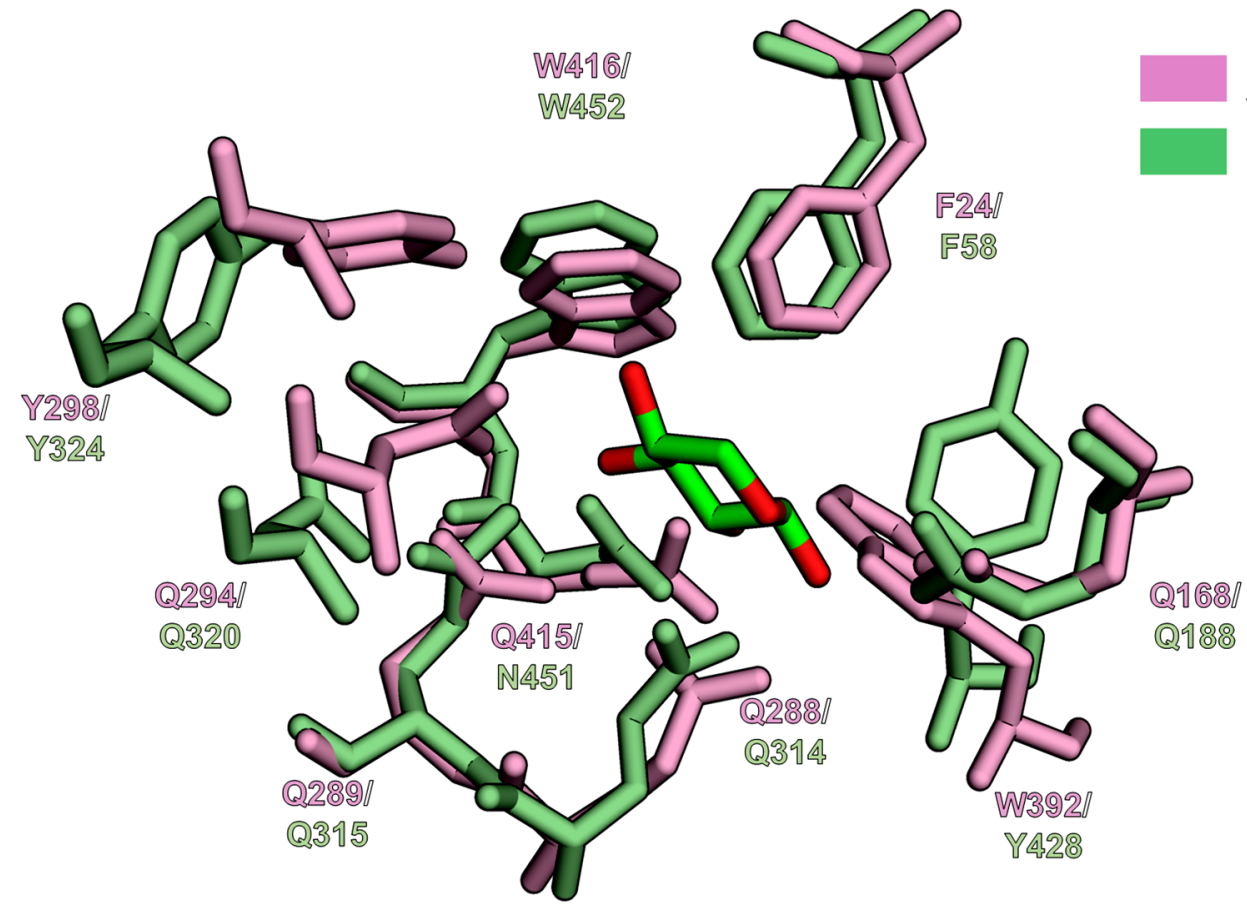

Fig. 6 Alignment of amino acid sequences of Cs4130, GXf1, STP10, XylE and GICPSE (a) and superposition of XyIE (pale pink) and Cs4130 (pale green) intermembrane residues related to xylose (green) transport (b). The residues mutated in XylE and their counterparts in Cs4130, Gxf1, STP10, and GICPSE are highlighted in green. The position of a-helix was signed based in XyIE. a, a-helix; ia, intracellular a-helix 
Table 2 Distance between the residue mutated in XylE to xylose (DtX) in Angstrom ( $\AA$ ) and the binding energies (kcal/ mol) of Cs4130, Gxf1, XylE and variants complexed to xylose through molecular docking analysis

\begin{tabular}{|c|c|c|c|c|}
\hline & XylE & Cs4130 & Gxf1 & $\operatorname{DtX}(\AA ̊)$ \\
\hline & \multicolumn{4}{|l|}{ Residue (kcal/mol) } \\
\hline Wild-type & -27.4 & -28.8 & -26.0 & - \\
\hline \multirow[t]{5}{*}{ Mut1 } & $F 24 A(-27.6 / 0.2)$ & F58A $(-27.8 / 1.0)$ & F58A (24.9/1.1) & 3.9 \\
\hline & $\underline{\mathrm{G} 83 \mathrm{~A}}$ & $\underline{\mathrm{G} 120 \mathrm{~A}}$ & $\underline{\mathrm{G} 120 \mathrm{~A}}$ & $\underline{22.0}$ \\
\hline & $\underline{\mathrm{R} 133 \mathrm{C}}$ & $\underline{\mathrm{R} 153 \mathrm{C}}$ & $\underline{\mathrm{R} 153 \mathrm{C}}$ & $\underline{11.7}$ \\
\hline & $\underline{E 153 \mathrm{~A}}$ & $\underline{E 173 A}$ & $\underline{E 173 A}$ & $\underline{17.8}$ \\
\hline & $\underline{\mathrm{R} 160 \mathrm{~A}}$ & $\underline{\mathrm{R} 180 \mathrm{~A}}$ & $\underline{\mathrm{R} 180 \mathrm{~A}}$ & $\underline{14.3}$ \\
\hline Mut 2 & Q168A (-28.3/0.9) & Q188A (-26.4/2.4) & $\mathrm{Q} 188 \mathrm{~A}(-25.5 / 0.5)$ & 2.3 \\
\hline Mut 3 & Q288A (-26.4/1.0) & Q314A (-28.1/0.7) & Q314A (-24.9/1.1) & 2.9 \\
\hline Mut 4 & Q289A (-26.6/0.8) & Q315A (-28.5/0.3) & Q315A (-26.1/0.1) & 3.0 \\
\hline Mut 5 & N294A (-28.1/0.7) & N320A (-28.2/0.6) & N320A $(-25.2 / 0.8)$ & 2.9 \\
\hline Mut 6 & Y298A (- 26.4/1.0) & Y324A (- 28.1/0.7) & Y324A (- 25.8/0.2) & 4.3 \\
\hline \multirow[t]{3}{*}{ Mut 7} & N325A $(-27.4 / 0)$ & N349A (- 26.9/1.9) & N349A $(-25.7 / 0.3)$ & $\underline{6.7}$ \\
\hline & $\underline{\mathrm{G} 340 \mathrm{~A}}$ & $\underline{\mathrm{G} 364 \mathrm{~A}}$ & $\underline{G} 364 \mathrm{~A}$ & $\underline{15.8}$ \\
\hline & $\underline{\mathrm{R} 341 \mathrm{~A}}$ & $\underline{\mathrm{R} 365 \mathrm{~A}}$ & $\underline{\mathrm{R} 365 \mathrm{~A}}$ & $\underline{14.3}$ \\
\hline \multirow[t]{3}{*}{ Mut 8} & W392A (- 26.6/0.8) & Y428A (- 27.9/0.9) & Y431A (- 25.6/0.7) & 3.1 \\
\hline & $\underline{E 397 A}$ & $\underline{E} 433 \mathrm{~A}$ & $\underline{\mathrm{E} 436 \mathrm{~A}}$ & $\underline{14.2}$ \\
\hline & $\underline{\mathrm{R} 404 \mathrm{~A}}$ & $\underline{\mathrm{K} 440 \mathrm{~A}}$ & $\underline{\mathrm{R} 443 \mathrm{~A}}$ & $\underline{16.1}$ \\
\hline Mut 9 & Q415A (-26.8/0.6) & N451A (-28.0/0.8) & N454A (- 26.6/0.6) & 2.7 \\
\hline Mut 10 & W416A (26.5/0.9) & W452A $(-28.5 / 0.3)$ & W455A (- 25.4/0.6) & 4.8 \\
\hline
\end{tabular}

Residues close and far from ligand (xylose) are highlighted in italics and underlined, respectively

The energy of binding $(\mathrm{kcal} / \mathrm{mol})$ of mutants and the difference from the wild-type are between parentheses

Mut mutant
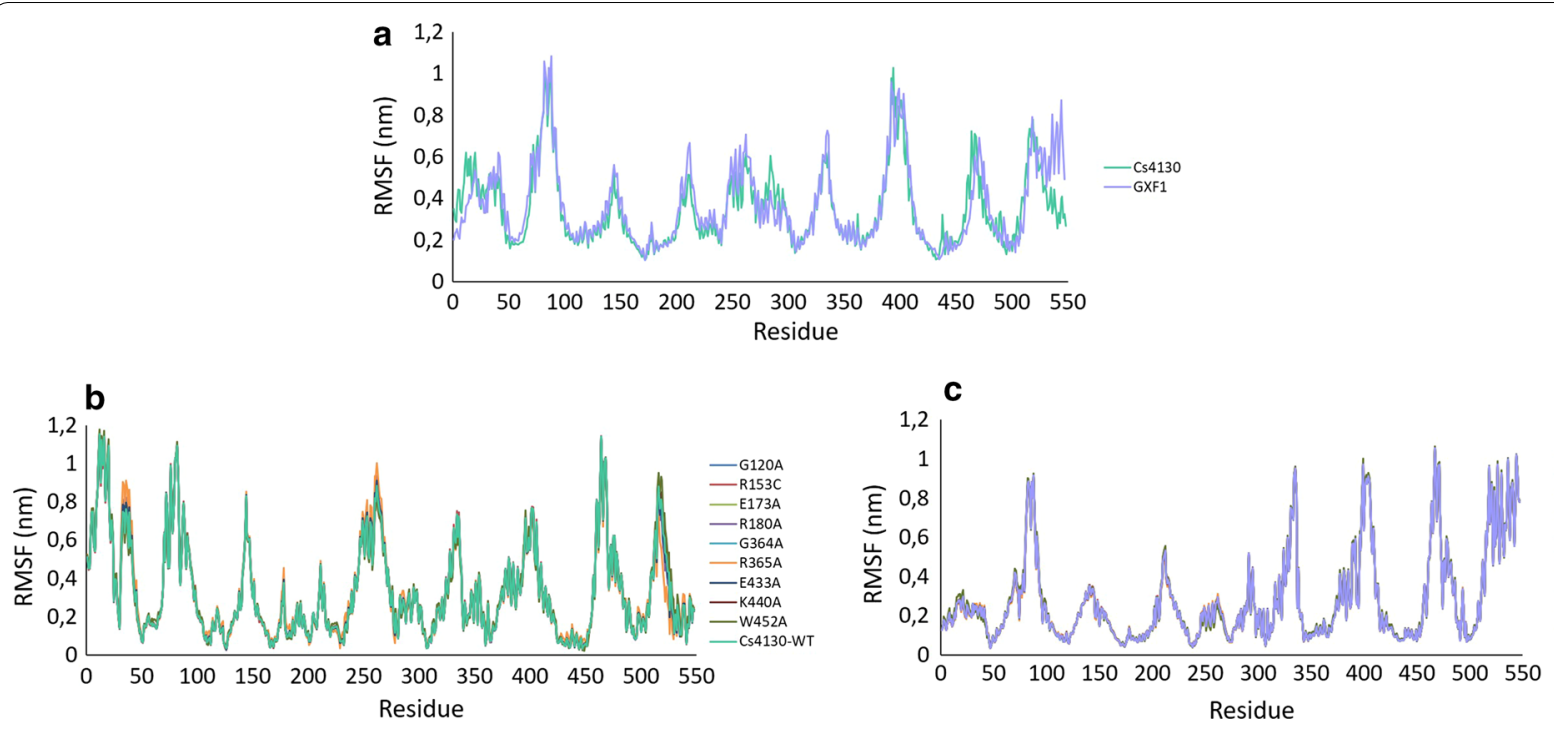

Fig. 7 Evaluation of the dynamical regime through non-linear normal mode analysis. Comparison between WTs Cs4130 and Gxf1 (a). The low-frequency mode 6 for Cs4130 and Gxf1 (with respective mutants) are shown in $\mathbf{b}$ and $\mathbf{c}$, respectively. The displacement of the residues was evaluated using the root mean square fluctuation (RMSF) 
WT. The residues G120, R153, E173, R180, G364, R365, E433 (E436 in Gxf1) and K440 (K443 in Gxf1) with distances from xylose underlined in Table 2, were mutated in Cs4130 and Gxf1 and their dynamical regimes were compared with the respective WTs. Figure $7 \mathrm{~b}$, c shows the RMSF average of Cs4130 and Gxf1, respectively. The mutation R365A in Cs4130 changes the dynamical behavior of Cs4130 WT, what is mostly evident in the low-frequency modes $6-8$ (Fig. $7 \mathrm{~b}$ shows only the mode 6), mainly in the residues $32-41$ (N-terminal), 257-263 (cytoplasmatic region) and 515-529 (C-terminal), probably resulting in altered xylose transport (Fig. 7b, Additional file 7: Figure S3, Additional file 8: Figure S4). For the other Cs4130 variants, the dynamical regimes were quite stable considering all low-frequency modes as also observed for Gxf1 (Fig. 7c).

\section{Discussion}

Metabolic engineering of microbial platforms for $2 \mathrm{G}$ biorefineries relies on the development of efficient transport systems to capture all the sugars from biomass [10, 12]. Xylose uptake across the plasma membrane is a critical limiting step in recombinant yeast strain development, reducing the efficiency of downstream pathways and conversion in high value-added bioproducts. Over the course of evolution, S. cerevisiae was highly equipped with a diverse and multipurpose transport system in order to deal with different conditions and challenging environments. The whole-genome duplication event expanded the repertoire of glucose transporters that $S$. cerevisiae has at its disposal and allows efficient growth in a wide range of glucose concentrations [45]. S. cerevisiae has 18 genes encoding hexose transporters-Hxt1p-Hxt17p and Gal2p, which belong to MFS. The endogenous transporters differ regarding the degree of glucose affinity, from low $\left(K_{\mathrm{m}}\right.$ of $\left.50-100 \mathrm{mmol} / \mathrm{L}\right)$ to high-affinity $\left(K_{\mathrm{m}}\right.$ of $\left.1-2 \mathrm{mmol} / \mathrm{L}\right)$, and are dependent on glucose concentration in the extracellular environment $[36,46]$. The transport system is also fine regulated at the transcriptional and post-translational levels to adapt to different glucose concentrations [47]. As xylose fermentation was probably not a strong selective pressure in S. cerevisiae evolution, engineered strains of S. cerevisiae do not harbor all those transport system options or a regulated signaling system that allows pentose uptake in a range of different concentrations. Using an in vivo biosensor panel associated with three major signaling pathways, a recent work showed that an engineered C5 $S$. cerevisiae strain senses xylose differently from glucose. The signal triggered at high concentrations of xylose was similar to the carbon starvation signal observed at low glucose levels $[48,49]$. In addition to the differences observed in cell signaling, in order to overcome transport limitations that result in low assimilation rates, a global modification at the xylose transport system is necessary with the integration of facilitators capable of transporting xylose in a wide range of sugar concentrations.

Natural evolution already resulted in many pentoseassimilating yeast species which transport xylose with high and low affinities and the functional expression of only a few heterologous transporters were demonstrated to improve xylose uptake in recombinant $S$. cerevisiae strains [21, 50-53]. For example, the xylose transporter Sut1 isolated from S. stipitis was able to improve xylose consumption by $25 \%$ and increase ethanol yield by $17 \%$ on recombinant S. cerevisiae cells [50]. The transporters Xyp29, AXT1, XYLH, and MGT05196 from C5 yeasts S. stipitis [52], Pichia guilliermondii [54], D. hansenii [55] and Meyerozyma guilliermondii [56], respectively, were also able to restore significant xylose growth on expressing strains. Even A. thaliana xylose transporters, At5g17010 and At5g59250, were responsible for 25\% and $40 \%$ of improvements in xylose consumption rate, respectively, on S. cerevisiae strains [57]. As highlighted above, the facilitator Gxf1 isolated from C. intermedia [34] has been described as the best heterologous xylose transporter, although it improved growth in xylose only at low sugar concentrations $(4 \mathrm{~g} / \mathrm{L})$, and no improvement was observed for concentrations higher than $15 \mathrm{~g} / \mathrm{L}$ [21, $26,33]$. Gxf1 shows an intermediate affinity to xylose and a glucose preference 20-fold higher [34].

By assessing microbiomes such as the digestive tract of plague insects and decayed biomasses, we isolated pentose yeast species capable of naturally using xylose (Additional file 2: Table S1). The cultivation in xylose by successive batches limited the naturally diverse population by substrate competition and allowed the isolation and molecular identification of only a few species able to consume xylose efficiently. Since these strains compete to grow in xylose, an efficient transport system to uptake the $\mathrm{C} 5$ sugar was expected. The yeast $C$. sojae was selected after comparative fermentations as a potential source of high-affinity xylose transporters. C. sojae was isolated from the intestine of a Coleoptera larvae collected internally from an energy cane stalk (Additional file 2: Table S1). From genome analysis [58], well-known eukaryotic xylose transporters, such as $H X T 7[20,22$, 59], GAL2 [20, 22], XUT1 [21], XUT3 [21], SUT1 [50, 51], GXS1 [28, 29, 34], GXF1 [25-27, 34], Xylhp [21] and other members from MFS were used as baits to select the transporter-encoding genes Cs186, Cs2608, Cs3894 and Cs4130. From phylogenetic comparative analysis (Fig. 1) two of the elect C. sojae transporters, Cs4130 and Cs3894 showed similarity to uniport transporters such as GXF1, HXT7 and GAL2, whereas Cs186 was related to XUT3 from S. stipitis. However, Cs2608 was more related to 
symporter transporters, such as GXS1. Therefore, these sequence similarities suggest that Cs186, Cs3894 and Cs4130 transporters are novel members of uniport transporters and Cs 2608 represents a new xylose/glucose $-\mathrm{H}^{+}$ symporter.

All selected C. sojae transporters were evaluated on EBY_Xyl1 strain, a modified version of EBY.VW4000 [39], expressing the XR/XDH/XKS xylose consumption pathway (Additional file 4: Table S2). C. sojae transporters were able to restore growth of EBY_Xyl1 in all sugars evaluated, showing a broader substrate specificity (Fig. 3). Regarding Cs186, its low xylose transport capacity may be explained not only by the distant relation to known xylose carriers but also by the presence of many identical copies on C. sojae genome (Cs186, Cs173, Cs183, Cs136) as shown in the polytomy in Fig. 2d. As transporters have a dose-dependent effect, this low capacity could be compensated by the extra copies, which together supply xylose uptake for C. sojae cells. Cs2608 is a symporter and only a few examples were functionally expressed on S. cerevisiae previously in the literature. Despite being functional and restoring EBY_Xyl1 growth, Cs2608 performed poorly on most of the tested sugars, especially glucose and mannose (Fig. 3).

The growth profile in xylose was different comparing $C$. sojae transporters and Gxf1 in crescent xylose concentrations (Fig. 4a). While the growth of Gxf1 strain decrease as the xylose concentration increase, strains expressing Cs3894 and Cs4130 showed superior xylose uptake at concentrations up to $50 \mathrm{~g} / \mathrm{L}$ (Figure 4a). Depending on the type of process used to release sugars from biomass, xylose concentrations in $2 \mathrm{G}$ hydrolysates can reach high values, with concentrations of $65 \mathrm{~g} / \mathrm{L}$ [60], $85 \mathrm{~g} / \mathrm{L}$ [61], and even higher values [62] already reported in the literature. Therefore, Cs3894 and Cs4130 are profitable candidates for xylose uptake due to their natural loss of inhibition at higher concentrations. Interestingly, Cs4130 restores the growth of recombinant strains even at high xylose concentrations, presenting an opposite behavior to Gxf1. Since Cs4130 enabled better growth compared to Cs3894, especially in higher concentrations of xylose, further experiments were done with only the first facilitator. The fermentation in liquid media confirmed the pattern of growth of Cs4130 observed in spot-assay experiments, presenting higher productivity in $50 \mathrm{~g} / \mathrm{L}$ against $10 \mathrm{~g} / \mathrm{L}$ of xylose. A more subtle difference between Cs4130 and Gxf1 was observed in fermentation in the liquid medium compared to growth in a solid medium, since transport differences may have been attenuated by limitations on the downstream pathway. EBY_Xyl1 cells expressing Cs4130 improved xylose consumption rates at $50 \mathrm{~g} / \mathrm{L}$ when compared to Gxf1 during liquid fermentation, while the opposite was observed at $10 \mathrm{~g} / \mathrm{L}$, maintaining the same consumption profile observed in the spot-assay experiment (Fig. 4b, c). The design of microbial cell factories to efficiently convert biomass sugars requires the introduction of transporters capable to assimilate xylose in different concentrations available in lignocellulosic hydrolysates. In this context, the combination of Gxf1 and Cs4130 to engineer a xylose-fermenting S. cerevisiae strain can be an interesting approach, assuring efficient xylose utilization in $2 \mathrm{G}$ hydrolysates.

Besides differences in transporter affinities, substrateinduced ubiquitination could also explain the differences observed between both transporters. Membrane transporter protein can be removed from the cytoplasmic membrane and degraded in the absence of glucose [63, 64]. For example, Hxt1 and Hxt3 are low-affinity transporters, expressed in the presence of a high concentration of glucose and degraded in the absence of glucose $[47,65,66]$. However, the molecular mechanisms that could explain if different concentrations of xylose trigger endocytosis are not clear and further investigation is required.

Simultaneous uptake of glucose and xylose is highly desirable for engineered 2G strains. Cells expressing a heterologous xylose assimilation pathway preferentially consume glucose and a diauxic growth is observed in hydrolysates. Glucose repression is caused mainly by competition for uptake through hexose transporters [67]. In order to investigate if the transport mechanism of Cs4130 alleviates the glucose inhibitory effect, we tested different growth conditions with mixtures of glucose and xylose. High-cell density fermentation with an equal concentration of both sugars (Fig. 5a), showed a preferential consumption of glucose in the first $10 \mathrm{~h}$, indicating a higher preference for glucose over xylose. We disrupted glycolysis in EBY_Xyl1_hx $k^{0}$, uncoupling glucose uptake from its metabolization, similarly as the strategy described by Farwick, et al. [31]. The mutant cell is unable to metabolize glucose when hexokinase genes (HXK1, HXK2 and GLK1) are knocked out [31, 68]. EBY_ Xyl1_hxk $k^{0}$ expressing GXF1 and Cs4130 showed strong glucose inhibition on a mixture of $\mathrm{C} 5$ and $\mathrm{C} 6$ (Fig. $5 \mathrm{~b}$ ). Higher glucose concentration on mixtures of $\mathrm{C} 5$ and $\mathrm{C} 6$ decreases the growth of the strain overall. A strong glucose inhibition was previously described for the native transporters Gal2p and Hxt7p using an $H X K$ null strain. In the same C5/C6 mixture condition using $10 \mathrm{~g} / \mathrm{L}$ of xylose and $50 \mathrm{~g} / \mathrm{L}$ of glucose, a severe impairment in xylose uptake was observed for both transporters, showing a higher affinity for glucose over xylose [31].

Sugar transport in cells is a dynamic event where transmembrane proteins oscillate between the outward/occluded and inward/occluded states. Some residues make the path for the ligand mainly by short-range 
interactions, for example, hydrogen bonds, which are constantly redesigned to pull the sugar into the cytoplasmatic face [69]. The sugar-binding regions in XylE [43], Cs4130 and Gxf1 are conserved. The differences in the binding energy values seen in Table 1 emphasize the specificities of each transporter when the same monosaccharide is taken into consideration (except fructose). Probably, the non-identical residues in the vicinity of the residues bound to sugar promote local changes in the environment affecting the interactions.

A comparison of XylE experimental data [43] with the molecular docking analysis of this work suggests that the increase/decrease of binding energies of variants equal or higher than $0.6 \mathrm{kcal} / \mathrm{mol}$ compared to their respective WTs seems to be enough to affect xylose transport. The only exception is the residue F24A in XylE (F58A in Cs4130 and Gxf1), where a difference in binding energy equal to $0.2 \mathrm{kcal} / \mathrm{mol}$ affect xylose transport (Table 2 ). The TM1 harbors the residues F24 and F58 in XylE and Cs4130/Gxf1, respectively (Fig. 6a). The conserved motif G-G/F-XXX-G in TM1 is vastly found in facilitators which capture xylose [28]. XylE, Cs4130 and Gxf1 present this conserved motif where the "XXX" are represented by L22/L23/F24 Y56/V57/F58 and F56/V57/ F58, respectively. Contrary to that observed for XylE, the mutation F40A (same position of F24 and F58 in XylE and Cs4130/Gxf1, respectively) in the transporter Gxs1 from $C$. intermedia increase the selectivity for xylose over glucose, being the best results attained for mutants F40M and F40S [28]. Also, mutation in the TM1 residue F79S (Y56 and F56 in Cs4130 and Gxf1, respectively) in Hxt7p favors the growth of $S$. cerevisiae in xylose as the sole carbon source and allows the co-consumption of xylose and glucose [70], revealing that a fine-tuning between the residues directly involved with the xylose transport and their vicinity should exist.

The potential contribution of residues distant to xylose for transport in Cs4130 and Gxf1 was evaluated by Normal Mode Analysis (NMA). NMA assumes that modes exhibiting the lowers frequencies describe the largest movements in a protein [71, 72]. The mutation R365A in Cs4130 changes the dynamical regimes mainly of the residues 32-41, 257-263 and 515-529 (Fig. 7b). A zoom view shows that residues adjacent to these ranges are also affected by R365A in a minor proportion (Additional file 7: Figure S3). Then, the variant R365A would probably result in a different transport phenotype for Cs4130, but not for Gxf1, which RMSF remains quite stable in all 10 low-frequency modes evaluated for all protein variants (Fig. 7c). It is important to emphasize that NMA uses simple constructions for the model, so small differences in the regime of fluctuations may be related to considerable variations in the dynamical behavior in physiological conditions. The residues affected by $\mathrm{R} 365 \mathrm{~A}$ are found mainly in the Cs4130 N and C-terminal regions. These portions, for both Cs4130 and Gxf1, do not fit with XylE crystallographic structure being promising sites to study the effect on sugar transport. Also, changes in the dynamical regimes of 257-263, intracellular face, could result in altered sugar transport given that loop regions are also important for the phenotype of transporters [36].

Some studies show that not only the residues directly involved in sugar binding are important for transport, but also those ones mapped far from sugar. Qureshi et al. [73] showed that polar interactions of residues about $15 \AA$ from sugar are determinant for the transport of glucose and fructose in PfHT1 from Plasmodium falciparum. The authors concluded that the substrate promiscuity is not devoid to sugar-binding site, but substrate-gating dynamics. In the same way, N367 in Hxt36 (N325 equivalent in XylE and N349 in Cs4130 and Gxf1) was mutated by all amino acids and the best results concerning S. cerevisiae growth in glucose and xylose were achieved with small and non-polar residues (mainly alanine and glycine) [74]. In XylE, N325A also retained the capacity to transport xylose [43].

The transport of sugars through the cellular membrane is not an obvious event in nature. Several bonds are made and broken in a restrictive and insoluble microenvironment. From the functional and in silico data, it is hypothesized that Cs4130 is a more promiscuous transporter than Gxf1 given it supports higher concentrations of xylose. In addition, mutations in Cs4130 will probably result in a diversity of responses not observed in Gxf1, for example, placing Cs4130 as a model to obtain transporters with improved properties regarding the assimilation of substrates other than glucose.

\section{Conclusions}

This work describes the novel xylose transporter Cs4130 and its functional expression in S. cerevisiae at high xylose concentrations, an important missing feature in C5 transporters previously described in the literature. The novel transporter was identified by accessing diverse microbiomes and using an evolutionary approach to prospect transporters candidates for industrial applications. The transport capacity of several targets was evaluated and Cs4130 stood out due to the natural loss of inhibition in high xylose concentrations. We also investigated the dynamic behavior of Cs4130 and conformational changes regarding xylose translocation, pointing out important residues related to transport in Cs4130 variants and revealing interesting aspects of xylose uptake mechanisms. These findings will be used for rational design procedures aiming for improved transport kinetics favoring 
the development of robust strains to face the challenges of 2 nd generation industries.

\section{Methods \\ Media and culture conditions}

Yeast cells were grown on liquid YP medium $(10 \mathrm{~g} / \mathrm{L}$ yeast extract and $20 \mathrm{~g} / \mathrm{L}$ peptone) supplemented with $20 \mathrm{~g} / \mathrm{L}$ $\mathrm{D}$-glucose (YPD) for cell propagation. For growth in xylose, D-glucose was replaced by $20 \mathrm{~g} / \mathrm{L} \mathrm{D}$-xylose (YPX). For transformants selection, cells were grown at $30{ }^{\circ} \mathrm{C}$ in complete synthetic media YNB (6.7 $\mathrm{g} / \mathrm{L}$ yeast nitrogen base without amino acids, Difco) supplemented with $1 \mathrm{~g} / \mathrm{L}$ drop-out without uracil, $20 \mathrm{~g} / \mathrm{L}$ glucose and $20 \mathrm{~g} / \mathrm{L}$ agar [75]. YP was autoclaved at $121{ }^{\circ} \mathrm{C}$ for $20 \mathrm{~min}$ and YNB was filter-sterilized using $0.2-\mu \mathrm{m}$ bottle-top filters. Strain EBY.VW4000, kindly provided by Prof. Eckhard Boles from Goethe university [39], and strain EBY_Xyl1 were grown in YNB with D-maltose instead of D-glucose, and EBY_Xyl1_ $h x k^{0}$ were cultivated in $3 \%$ ethanol as carbon source. EBY_Xyl1 expressing transporter genes were grown in solid YNB supplemented with different carbon sources (D-galactose, D-mannose, D-fructose, D-xylose, D-glucose and D-maltose) for spot-assay analysis. High-cell density fermentation of EBY_Xyl1 expressing the heterologous transporters were performed on YNB supplemented with $5 \mathrm{~g} / \mathrm{L}$ of casamino acids (Difco), $1 \mathrm{~g} / \mathrm{L}$ of tryptophan (Sigma) and the correspondent carbon source, D-maltose, D-xylose or D-glucose. The yeast cells isolation media was adapted from Cadete et al. [76]: $6.7 \mathrm{~g} / \mathrm{L}$ yeast nitrogen base without amino acids (Difco) supplemented with $1 \mathrm{~g} / \mathrm{L}$ of complete drop-out, $7 \mathrm{~g} / \mathrm{L}$ of D-xylose, $0.2 \mathrm{~g} / \mathrm{L}$ of chloramphenicol. For solid media, $10 \mathrm{~g} / \mathrm{L} \mathrm{D}$-xylose and $20 \mathrm{~g} / \mathrm{L}$ agar were employed. The selection media for strains with pSH65 plasmid was supplemented with $300 \mu \mathrm{g} / \mathrm{mL}$ of zeocin (Thermo Scientific) on solid plates. For URA3/5-FOA counter-selection procedure, strains expressing $\mathrm{pSH} 65$ plasmid were grown in synthetic medium supplemented with $440 \mathrm{mg} / \mathrm{mL}$ of uracil and $1 \mathrm{~g} / \mathrm{L}$ of 5-FOA (Zymo Research). For antibiotic resistance, $200 \mathrm{mg} / \mathrm{L}$ of geneticin and $200 \mathrm{mg} / \mathrm{L}$ of hygromycin was added to YPD for strains expressing the KanMX4 (G418) [77] or hph [78] markers, respectively. Likewise, $300 \mu \mathrm{g} / \mathrm{mL}$ of zeocin was added for strains transformed with pSH65 [79]. Permanent stocks of yeasts and $E$. coli cells were mixed with glycerol before storage at $-80{ }^{\circ} \mathrm{C}$.

\section{Isolation and screening of $\mathrm{D}$-xylose-consuming yeasts}

The C5-yeasts were isolated from two different sites: São Miguel dos Campos, Alagoas, Brazil, and Cosmópolis, São Paulo, Brazil. Samples from decayed energy cane and sugarcane and pest insects associated with this material were collected from the former location. Pest insects isolated from corn were isolated from the latter location. Decayed energy cane and sugarcane samples were collected closest to an ethanol mill, where the material was exposed to the environment for a long period. The local weather is hot and humid, with an average temperature from 20 to $32{ }^{\circ} \mathrm{C}$ and average annual precipitation of $1408 \mathrm{~mm}$. Twenty samples of each material were collected in July 2015, stored in sterile recipients and transported under refrigeration to the laboratory in less than $24 \mathrm{~h}$, according to Cadete et al. [76]. The same procedure was adopted for plague insects collected from plant material. For sugarcane and energy cane, $1 \mathrm{~g}$ of each sample was placed separately in $125 \mathrm{~mL}$ erlenmeyer flasks containing $50 \mathrm{~mL}$ of isolation xylose medium. For insects' samples, guts were removed in a sterile environment and macerated with a micropistil in a $1.5 \mathrm{~mL}$ conical tube containing $400 \mu \mathrm{L}$ of isolation medium, and then re-suspended separately in $125 \mathrm{~mL}$ erlenmeyer flasks containing $50 \mathrm{~mL}$ of isolation xylose medium. The flasks were incubated for 2-6 days in orbital shakers at $27^{\circ} \mathrm{C}, 150 \mathrm{rpm}$ until growth was visually detected. Five consecutive batches were carried out in the same medium to promote population enrichment and selection of strains capable of assimilating xylose rapidly. After growth, $50 \mu \mathrm{L}$ of each medium was spread with Drigalski spatula in Petri dishes containing xylose isolation solid medium. Plates were incubated at $30^{\circ} \mathrm{C}$ until colonies were observed. The isolated yeasts were maintained in permanent frozen stocks using 30\% glycerol solution at $-80{ }^{\circ} \mathrm{C}[75]$.

\section{DNA extraction and molecular identification}

C5-yeast species were identified using the internal transcribed spacer sequence (ITS1-5.8S-ITS2) and D1/ D2 domains. DNA extraction was performed using the standard S. cerevisiae protocol described in Ausubel [75]. The ITS1-5.8S-ITS2 and D1/D2 sequences were amplified by PCR using GoTaq ${ }^{\circledR}$ DNA polymerase (Promega) as described in FELL et al. [80] and Cadete et al. [76]. PCR amplicons were sequenced in a Sanger platform using an Applied Biosystems ${ }^{\circledR}$ Genetic Analyzer 3500. The amplicons were manually trimmed using a base QV of 20 and grouped in contigs. These contigs were used in BLASTn megablast program at the NCBI database (http://blast.ncbi.nlm.nih.gov/Blast.cgi). The ITS and D1/ D2 domains were retrieved from type material (CBShttp://www.cbs.knaw.nl/) of the top five hits in BLASTn and the sequences were globally aligned against the used query utilizing Clustal W in MEGA version 6 [81]. The query that had $<1 \%$ divergence to material type sequence, was considered from the same species of the type strain [82]. 


\section{Comparative fermentation of $\mathrm{C} 5$-yeasts}

In order to select the most suitable source of genes related to xylose metabolism, yeasts isolated and molecularly identified were compared in YPX medium. The ability to ferment D-xylose was screened using Durham tubes and the yeast S. stipitis NRRL Y-7124 as a positive control. Tubes were incubated at $30^{\circ} \mathrm{C}$ on shaker at $150 \mathrm{rpm}$ for 10 days to observe gas production. The ability to assimilate xylose of the isolated yeast species was also compared in erlenmeyer with $150 \mathrm{~mL}$ of YPX $(30 \mathrm{~g} / \mathrm{L})$ in orbital shakers, incubated at $30{ }^{\circ} \mathrm{C}$ and $150 \mathrm{rpm}$, starting with an optical density at $600 \mathrm{~nm}$ of approximately 1.0. All experiments were conducted in triplicates and samples were collected to measure optical density and to quantify metabolites with HPLC.

\section{Analytical procedures}

Samples from fermentation were analyzed regarding glucose, xylose, ethanol, glycerol, xylitol, succinic acid and acetic acid concentrations using a Waters e2795 HPLC with an Hpx 84 h column (Biorad) A standard curve with known concentrations of the compounds highlighted above was also analyzed using the same procedure. Ethanol, xylitol and glycerol yields (Yp/s, g/g) were calculated considering the ratio of the specific product formation to sugar consumption. Productivity $(\mathrm{Qp}, \mathrm{g} / \mathrm{L} \mathrm{h})$ was calculated as the ratio of product concentration $(\mathrm{g} / \mathrm{L})$ and fermentation time (h).

\section{Phylogenetic analysis}

The ability to ferment xylose for yeast species used in the phylogenetic analysis was assumed using the description of type strains deposited in Centraalbureau voor Schimmelcultures (CBS-http://www.cbs.knaw.nl/) yeast collection. We chose and downloaded a wide phylogenetic range of public yeast species genomes in Joint Genome Institute (JGI) Mycocosm from https://mycoc osm.jgi.doe.gov/mycocosm/species-tree/tree;FrDK5 q? organism=ascomycota. A large comparative analysis of these genomes was done by our group previously [37]. From the previous analysis, homologous gene families appeared. Orthology prediction was made using Markov clustering as implemented in OrthoMCL [83] which clusters orthologs and paralogs per similarity in functional groups called hereafter as gene families or orthogroups. The distribution of these families obeyed a numerical descending order, where larger families come before smaller families, being Fam0 the largest one. Transporter gene families were obtained using known xylose transporter proteins as baits in a BLAST search against gene families (Additional file 9: Table S5).

Assuming that all putative transporter gene families are homolog as a group, we proceeded with a gene phylogenetic tree reconstruction using all families and some outgroup sequences from bacteria and plants (Fig. 1). Amino acids sequences were globally aligned using MAFFT [84]. A maximum likelihood tree inference was carried in IQTree using its own adjustment substitution model inference (BIC and AIC criteria implemented) and 1000 bootstraps for branch support.

\section{Plasmid assembly}

Expression cassettes and plasmids were assembled in a single reaction using the Gibson Assembly ${ }^{\circledR}$ Master Mix (New England Biolabs-NEB) [85], and the backbone pRS426 [86] cleaved with BamHI (Promega). The sequences coding the putative transporters were amplified by PCR using Phusion DNA polymerase (New England BioLabs-NEB) from the gDNA of C. sojae. The control transporter GXF1 was amplified from Candida intermedia genome, using specific primers for each gene (Additional file 10: Table S6). Sequences of TDH1 promoter and terminator were amplified from $S$. cerevisiae strain LVA1 genome [10]. Fragments from PCR amplifications were purified from agarose gel using the Kit Wizard $^{\circledR}$ SV Gel and PCR Clean-Up System (Promega) before the assembly reaction. Cassettes containing the genes XYL1 and XYL2 from S. stipitis and XKS1 from S. cerevisiae were amplified from the plasmids pSsXRXDH and pScXKS (Santos, personal communication). Both cassettes use the URA3 gene flanked by two loxP sites as marker. The correct frame of the gene sequences cloned in the plasmids and expression cassettes were confirmed by Sanger sequencing. The main components of the plasmids are summarized in Additional file 11: Table S7.

\section{Construction of Saccharomyces cerevisiae strains}

The molecular techniques were performed using standard procedures [75]. The $S$. cerevisiae strains, primers and plasmids used in this study are listed in Additional files 4, 10, 11 Tables S2, S6 and S7, respectively. Expression cassettes containing the genes $X Y L 1, X Y L 2$ and $X K S 1$ were amplified by PCR, purified from agarose gel using the kit Wizard $^{\circledR}$ SV Gel and PCR Clean-Up System (Promega) and used to transform EBY.VW4000 strain using the LiAc/SS-DNA/PEG protocol [87]. The stable integration of the two expression cassettes was molecularly verified. The auxotrophic marker URA3 was excised using the plasmid pSH65 (Cre-loxP system) [79]. The Cre-recombinase enzyme, used to restore uracil auxotrophy, was induced when strains were grown in maltose. Counterselection was performed using 5-FOA to select strains that excised URA3. Transformants of EBY_Xyl1 harboring the plasmids pCIGXF1, pCS186, pCS2608, pCS3894, pCS4130 and pRS426 were selected in YNB medium lacking uracil. The transformation was confirmed by 
PCR. Deletion of the hexokinase genes $H X K 1, H X K 2$ and GLK1 was carried out by insertion of the antibiotic markers hph, KanMX4 and ble, amplified from pAG32 [78], pFA6KANMX4 [77] and pSH65 [79], respectively, resulting in EBY_Xyl1_ $h x k^{0}$ mutant cell (Additional file 4: Table S2).

\section{Microscopy of GFP-tagged strains}

Codon-optimized monomeric Green Fluorescent Protein-GFP mUkG1 from soft coral [88] was synthesized by Genscript and fused to the transporter sequence in the plasmids. Strain EBY_Xyl1 harboring the Cs4130mUkG1 was grown on YNB without uracil, supplemented with maltose $20 \mathrm{~g} / \mathrm{L}$ overnight at $30^{\circ} \mathrm{C} / 200 \mathrm{rpm}$. Yeast cells were examined by confocal fluorescence microscopy (Leica TCS SP8 inverted microscope). The GFP emitted fluorescence was captured by a Hybrid Detector set to the $\lambda$-range of 503-530 $\mathrm{nm}$. Differential Interference Contrast (DIC) images were also captured to show yeast morphology. Data were analyzed in LAS X core software (Leica microsystems).

\section{Spot growth assay}

The growth of the EBY_Xyl1 mutant strains were evaluated by spot assay as previously described [31]. The yeast cells were cultured in YNB without uracil, supplemented with maltose $20 \mathrm{~g} / \mathrm{L}$ for $24 \mathrm{~h}$ at $30^{\circ} \mathrm{C} / 200 \mathrm{rpm}$. Then, the cells were harvested by centrifugation at $3000 \mathrm{rpm}$ for $5 \mathrm{~min}$, washed three times and resuspended in water to $\mathrm{OD}_{600}=1.0$. Using an automatic multichannel pipette, $5 \mu \mathrm{L}$ of a tenfold serial dilution of the cells were spotted on Petri dishes with YNB synthetic complete medium without uracil and different carbon sources $(20 \mathrm{~g} / \mathrm{L}$ : maltose (control), glucose, galactose, xylose, mannose or fructose). The growth of EBY_Xyl1 strain was also evaluated in YNB containing 5, 10, 30 and $50 \mathrm{~g} / \mathrm{L}$ of xylose in order to evaluate if the strain growth is sugar concentration-dependent. The cells were incubated at $30{ }^{\circ} \mathrm{C}$ for 3 days (maltose), 5 days (glucose, galactose, mannose and fructose) and 7 days (xylose). All the experiments were performed in triplicate and repeated at least twice. For EBY_Xyl1_hxk ${ }^{0}$ drop assay, the cells were inoculated in $50 \mathrm{~mL}$ erlenmeyer flasks with YNB medium without uracil, supplement with $3 \%$ ethanol for $56 \mathrm{~h}$ at $30{ }^{\circ} \mathrm{C} / 200 \mathrm{rpm}$. YNB was supplemented with $10 \mathrm{~g} / \mathrm{L}$ of xylose and glucose (1:1), $10 \mathrm{~g} / \mathrm{L}$ of xylose and $20 \mathrm{~g} / \mathrm{L}$ of glucose (1:2) and $10 \mathrm{~g} / \mathrm{L}$ of xylose and $50 \mathrm{~g} / \mathrm{L}$ of glucose (1:5). Strains grown in YNB containing glucose $20 \mathrm{~g} / \mathrm{L}$, ethanol $3 \%$ or xylose $10 \mathrm{~g} / \mathrm{L}$ were adopted as controls. All experiments were performed in triplicate and the cultures were incubated for 10 days.

\section{Fermentation assays}

In order to confirm the Cs4130 behavior in different concentrations of sugar, fermentations were conducted in liquid media. The yeast strains were pre-grown on YNB synthetic medium (without uracil) supplemented with $5 \mathrm{~g} / \mathrm{L}$ of casamino acids and $50 \mathrm{~g} / \mathrm{L}$ of D-maltose until reach the stationary phase. Then, the cells were harvested by centrifugation, washed three times with sterile water and resuspended to an $\mathrm{OD}_{600}$ of 20 . The fermentation experiments were performed using $60 \mathrm{~mL}$ of YNB supplemented with $5 \mathrm{~g} / \mathrm{L}$ of casamino acids, $10 \mathrm{~g} / \mathrm{L}$ and $50 \mathrm{~g} / \mathrm{L}$ of xylose. For simultaneous glucose and xylose cofermentation, $10 \mathrm{~g} / \mathrm{L}$ of both sugars were used. The cells were incubated at $30{ }^{\circ} \mathrm{C} / 200 \mathrm{rpm}$. The experiments were performed in triplicate and samples were collected to measure optical density and for HPLC analysis.

\section{Docking and normal mode analysis}

Molecular Docking calculations were performed using UCSF Dock 6.9 [89]. The input files were prepared with UCSF Chimera 1.13.1 [90]. Cs4130 and Gxf1 tridimensional structures were modeled in I-TASSER web server [42]. The models were constructed based on the crystallographic structure of XylE (PBD code: 4GBY) complexed with $\mathrm{D}$-xylose $\quad(\mathrm{TM}-\mathrm{Score}=0.774)$, which indicates the most suitable regions for docking runs. 4GBY was employed as a reference to define the best force-field for sugar interactions based on previous reports of the influence of XylE variants for xylose transport [43]. The ligands were extracted from HIC-Up database (http:// xray.bmc.uu.se/hicup/). Receptors and ligands were prepared using Dock Prep tool and the potential binding pockets were detected with Sphgen tool. Then, the closest cavities to D-xylose in XylE were chosen. Charges were added through UCSF Chimera using AMBER ss12sb and AM1-BCC force-fields for standard and nonstandard residues, respectively. The molecular docking was carried out through Anchor and Grow algorithm and the energy of interaction was calculated with GridScore function due to the best agreement with the XylE mutants' experimental results. Ligand, receptor, ligands orientation and overlap bins were set to $0.2 \AA$ and the distance form matching among the atoms and receptor were set to $0.75 \AA$. The number of conformations evaluated per run (a total of 300 independent runs) was 500. The conformation with the lowest energy interaction value was selected. The effect of mutations in Cs4130 and Gxf1 were evaluated by two independent methods: Molecular Docking calculations and normal mode analysis (NMA). Residues close to xylose $(<5 \AA)$ were evaluated through docking analysis since they may be directly related to transport. NMA otherwise can quickly and extensively 
evaluate how mutations could affect the protein fluctuation regime or indicate allosteric effects. Non-linear NMA were performed with NOLB software [44] by generating 20 samples and evaluating the 10 lowest frequencies through root-mean square fluctuation (RMSF) using GROMACS toolkit [91].

\section{Supplementary information}

Supplementary information accompanies this paper at https://doi. org/10.1186/s13068-020-01782-0.

Additional file 1: Figure S1. Microbiomes exploited for the isolation of wild C5-yeasts. A. Termite associated with lignocellulosic material; B. Vessel formed by insect pest in energy cane; $C$. Sugarcane in a state of decomposition; D. Coleoptera larvae isolated from energy cane.

Additional file 2: Table S1. Fermentation performance of isolated C5 yeast species in YPX $20 \mathrm{~g} / \mathrm{L}$. Yield (Yp/s) is expressed as grams of product (gp) per grams of xylose. Xylose consumption was measured after $54 \mathrm{~h}$ of fermentation. C5 yeasts isolated from decayed energy cane (EC), sugarcane (SC), sugarcane straw (SS), corn crops (CC) and associated insect pests.

Additional file 3: Figure S2. C. sojae fermentation of xylose as the sole carbon source. C. sojae were cultivated in YPX (30 g/L) in batch fermentation with a low initial optical density of 0.5 . Fermentation assays were performed in triplicate and error bars represent the standard deviation from the average values.

Additional file 4: Table S2. S. cerevisiae strains used in the study. Additional file 5: Table S3. Variants of XyIE [43] and equivalent residues in CS4130, Gxf1, STP10 [92] and GICP ${ }_{\text {SE }}$ [93].

Additional file 6: Table S4. Binding energies (kcal/mol) of Cs4130, Gxf1 mutants complexed with xylose through molecular docking analysis.

Additional file 7: Figure S3. Zoom inset of the main amino acids in Cs4130 (32-41, 257-263 and 515-529) with the dynamical regimes affected by the mutation R365A.

Additional file 8: Figure S4. Evaluation of the dynamical regimes of Cs4130 and Gxf1 through non-linear Normal Mode Analysis. The average of all low-frequencies for Cs4130 and Gxf1 is presented in A and B, respectively. The displacement of the residues was evaluated using the root mean square fluctuation (RMSF).

Additional file 9: Table S5. Xylose transporter proteins used in phylogenetic analysis.

Additional file 10: Table S6. Primers used in this study. Homology to promoter (Pr) and terminator (Ter) TDH1 are shown in bold.

Additional file 11: Table S7. Plasmids used in the study.

\section{Abbreviations}

2G: Second-generation; MFS: Major facilitator superfamily; XR: Xylose reductase; XDH: Xylitol dehydrogenase; XKS1: Xylulokinase; HXK: Hexokinase; 5-FOA: 5-Fluoroorotic acid; ITS: Internal transcribed spacer; NMA: Normal mode analysis; RMSF: Root-mean square fluctuation; UPR: Unfolded protein response.

\section{Acknowledgements}

We are thankful to Centro Nacional de Pesquisas em Energia e Materiais (CNPEM), Fundação de Amparo à Pesquisa do Estado de São Paulo (FAPESP, Grant Numbers: 2017/08519-6, 2017/05078-9 and 2018/00888-5), Conselho Nacional de Desenvolvimento Científico e Tecnológico (Grant Number: 430291/2018-3) and Serrapilheira Institute (Grant Number: Serra-1708-16205). This study was financed in part by the Coordenação de Aperfeiçoamento de Pessoal de Nível Superior-Brasil (CAPES)_Finance Code 001. We also thank Prof. Eckhard Boles from Goethe University for kindly provide EBY.VW4000 strain. Simulations were performed at CENAPAD-SP (Centro Nacional de
Processamento de Alto Desempenho em São Paulo), UNICAMP/FINEP- MCT project.

\section{Authors' contributions}

JGRB performed the molecular biology/fermentation assays and wrote the manuscript, GB isolated the yeast cells from diverse microbiomes, TLRC and LCO performed and analyzed the structural models, docking and molecular dynamics data, MG and JJ performed the phylogenetic analysis, MC performed the microscopy analysis, GAGP supervised the work and revised the manuscript, LVS designed, supervised, wrote and revised the manuscript. All authors read and approved the final manuscript.

\section{Funding}

This work was supported by Fundação de Amparo à Pesquisa do Estado de São Paulo (FAPESP, Grant Numbers: 2017/08519-6, 2017/05078-9, 2018/008885), Conselho Nacional de Desenvolvimento Científico e Tecnológico (Grant Number: 430291/2018-3) and Serrapilheira Institute (Grant Number: Serra1708-16205). This study was financed in part by the Coordenação de Aperfeiçoamento de Pessoal de Nível Superior-Brasil (CAPES)_Finance Code 001.

\section{Availability of data and materials}

All data generated or analyzed during this study are included in this published article and its additional files.

\section{Ethics approval and consent to participate}

Not applicable.

\section{Consent for publication}

Not applicable.

\section{Competing interests}

The authors declare that they have no competing interests.

\section{Author details}

${ }^{1}$ Brazilian Biorenewable National Laboratory (LNBR), Brazilian Center for Research in Energy and Materials (CNPEM), Campinas, São Paulo 13083-100, Brazil. ${ }^{2}$ Genetics and Molecular Biology Graduate Program, Institute of Biology, University of Campinas (UNICAMP), Campinas, Brazil. ${ }^{3}$ Brazilian Biosciences National Laboratory (LNBio), Brazilian Center for Research in Energy and Materials (CNPEM), Campinas, Sao Paulo 13083-970, Brazil. ${ }^{4}$ Brazilian Synchrotron Light Laboratory (LNLS), Brazilian Center for Research in Energy and Materials (CNPEM), Campinas, Sao Paulo 13083-970, Brazil. ${ }^{5}$ Department of Physics-Institute of Biosciences, Humanities and Exact Sciences, UNESP, São Paulo State University, São José do Rio Preto, São Paulo 15054-000, Brazil.

Received: 18 May 2020 Accepted: 4 August 2020

Published online: 14 August 2020

\section{References}

1. Naik SN, Goud VV, Rout PK, Dalai AK. Production of first and second generation biofuels: a comprehensive review. Renew Sustain Energy Rev. 2010;14:578-97.

2. Nanda S, Azargohar R, Dalai AK, Kozinski JA. An assessment on the sustainability of lignocellulosic biomass for biorefining. Renew Sustain Energy Rev. 2015;50:925-41.

3. Lee RA, Lavoie J-M. From first- to third-generation biofuels: challenges of producing a commodity from a biomass of increasing complexity. Anim Front. 2013;3:6-11.

4. Nigam PS, Singh A. Production of liquid biofuels from renewable resources. Prog Energy Combust Sci. 2011;37:52-68.

5. Jordan DB, Bowman MJ, Braker JD, Dien BS, Hector RE, Lee CC, Mertens JA, Wagschal K. Plant cell walls to ethanol. Biochem J. 2012;442:241-52.

6. dos Santos LV, de Barros Grassi MC, Gallardo JCM, Pirolla RAS, Calderón $\mathrm{LL}$, de Carvalho-Netto OV, et al. Second-generation ethanol: the need is becoming a reality. Ind Biotechnol. 2016:12:40-57.

7. Nielsen J, Keasling JD. Engineering cellular metabolism. Cell. 2016:164:1185-97. 
8. Lu H, Li F, Sánchez BJ, Zhu Z, Li G, Domenzain I, et al. A consensus S. cerevisiae metabolic model Yeast8 and its ecosystem for comprehensively probing cellular metabolism. Nat Commun. 2019;10:3586.

9. Choi KR, Jang WD, Yang D, Cho JS, Park D, Lee SY. Systems metabolic engineering strategies: integrating systems and synthetic biology with metabolic engineering. Trends Biotechnol. 2019;37:817-37.

10. dos Santos LV, Carazzolle MF, Nagamatsu ST, Sampaio NMV, Almeida LD, Pirolla RAS, et al. Unraveling the genetic basis of xylose consumption in engineered Saccharomyces cerevisiae strains. Sci Rep. 2016;6:38676.

11. Long CP, Antoniewicz MR. How adaptive evolution reshapes metabolism to improve fitness: recent advances and future outlook. Curr Opin Chem Eng. 2018;22:209-15.

12. Verhoeven MD, Lee $M$, Kamoen $L$, van den Broek M, Janssen DB, Daran J-MG, van Maris AJ, Pronk JT. Mutations in PMR1 stimulate xylose isomerase activity and anaerobic growth on xylose of engineered Saccharomyces cerevisiae by influencing manganese homeostasis. Sci Rep. 2017;7:46155.

13. Lee $\mathrm{S}-\mathrm{M}$, Jellison T, Alper HS. Systematic and evolutionary engineering of a xylose isomerase-based pathway in Saccharomyces cerevisiae for efficient conversion yields. Biotechnol Biofuels. 2014;7:122.

14. Quistgaard EM, Löw C, Guettou F, Nordlund P. Understanding transport by the major facilitator superfamily (MFS): structures pave the way. Nat Rev Mol Cell Biol. 2016;17:123-32.

15. Marger MD, Saier MH. A major superfamily of transmembrane facilitators that catalyse uniport, symport and antiport. Trends Biochem Sci. 1993;18:13-20.

16. Henderson PJF, Baldwin SA. This is about the in and the out. Nat Struct Mol Biol. 2013;20:654-5.

17. Pao SS, Paulsen IT, Saier MH. Major facilitator superfamily. Microbiol Mol Biol Rev. 1998;62:1-34.

18. Forrest $L R$, Krämer $R$, Ziegler $C$. The structural basis of secondary active transport mechanisms. Biochim Biophys Acta Bioenerg. 2011;1807:167-88.

19. Reddy VS, Shlykov MA, Castillo R, Sun El, Saier MH. The major facilitator superfamily (MFS) revisited. FEBS J. 2012;279:2022-35.

20. Sedlak M, Ho NWY. Characterization of the effectiveness of hexose transporters for transporting xylose during glucose and xylose co-fermentation by a recombinant Saccharomyces yeast. Yeast. 2004;21:671-84.

21. Young E, Poucher A, Comer A, Bailey A, Alper H. Functional survey for heterologous sugar transport proteins, using Saccharomyces cerevisiae as a host. Appl Environ Microbiol. 2011;77:3311-9.

22. Hamacher T, Becker J, Gárdonyi M, Hahn-Hägerdal B, Boles E. Characterization of the xylose-transporting properties of yeast hexose transporters and their influence on xylose utilization. Microbiology. 2002;148:2783-8.

23. Maier A, Völker B, Boles E, Fuhrmann GF. Characterisation of glucose transport in Saccharomyces cerevisiae with plasma membrane vesicles (countertransport) and intact cells (initial uptake) with single Hxt1, Hxt2, Hxt3, Hxt4, Hxt6, Hxt7 or Gal2 transporters. FEMS Yeast Res. 2002;2:539-50.

24. Aeling KA, Salmon KA, Laplaza JM, Li L, Headman JR, Hutagalung AH, et al. Co-fermentation of xylose and cellobiose by an engineered Saccharomyces cerevisiae. J Ind Microbiol Biotechnol. 2012;39:1597-604.

25. Runquist D, Fonseca C, Rådström P, Spencer-Martins I, Hahn-Hägerdal B. Expression of the Gxf1 transporter from Candida intermedia improves fermentation performance in recombinant xylose-utilizing Saccharomyces cerevisiae. Appl Microbiol Biotechnol. 2009:82:123-30.

26. Fonseca C, Olofsson K, Ferreira C, Runquist D, Fonseca LL, Hahn-Hägerdal $\mathrm{B}$, et al. The glucose/xylose facilitator Gxf1 from Candida intermedia expressed in a xylose-fermenting industrial strain of Saccharomyces cerevisiae increases xylose uptake in SSCF of wheat straw. Enzyme Microb Technol. 2011:48:518-25.

27. Leandro MJ, Spencer-Martins I, Gonçalves P. The expression in Saccharomyces cerevisiae of a glucose/xylose symporter from Candida intermedia is affected by the presence of a glucose/xylose facilitator. Microbiology. 2008;154:1646-55.

28. Young EM, Tong A, Bui H, Spofford C, Alper HS. Rewiring yeast sugar transporter preference through modifying a conserved protein motif. Proc Natl Acad Sci USA. 2014;111:131-6.

29. Young EM, Comer AD, Huang H, Alper HS. A molecular transporter engineering approach to improving xylose catabolism in Saccharomyces cerevisiae. Metab Eng. 2012;14:401-11.
30. Bracher JM, Verhoeven MD, Wisselink HW, Crimi B, Nijland JG, Driessen AJM, et al. The Penicillium chrysogenum transporter PcAraT enables high-affinity, glucose-insensitive L-arabinose transport in Saccharomyces cerevisiae. Biotechnol Biofuels. 2018;11:63.

31. Farwick A, Bruder S, Schadeweg V, Oreb M, Boles E. Engineering of yeast hexose transporters to transport D-xylose without inhibition by D-glucose. Proc Natl Acad Sci USA. 2014;111:5159-64.

32. Kim SR, Ha SJ, Wei N, Oh EJ, Jin YS. Simultaneous co-fermentation of mixed sugars: a promising strategy for producing cellulosic ethanol. Trends Biotechnol. 2012;30:274-82.

33. Runquist D, Hahn-Hagerdal B, Radstrom P. Comparison of heterologous xylose transporters in recombinant Saccharomyces cerevisiae. Biotechnol Biofuels. 2010;3:5

34. Leandro MJ, Gonçalves P, Spencer-Martins I. Two glucose/xylose transporter genes from the yeast Candida intermedia: first molecular characterization of a yeast xylose-H+ symporter. Biochem J. 2006;395:543-9.

35. Parachin NS, Bergdahl B, van Niel EWJ, Gorwa-Grauslund MF. Kinetic modelling reveals current limitations in the production of ethanol from xylose by recombinant Saccharomyces cerevisiae. Metab Eng. 2011;13:508-17.

36. Reznicek O, Facey SJ, de Waal PP, Teunissen AWRH, de Bont JAM, Nijland JG, Driessen AJM, Hauer B. Improved xylose uptake in Saccharomyces cerevisiae due to directed evolution of galactose permease Gal2 for sugar co-consumption. J Appl Microbiol. 2015;119:99-111.

37. Borelli G, Fiamenghi MB, Dos Santos LV, Carazzolle MF, Pereira GAG, José J. Positive selection evidence in xylose-related genes suggests methylglyoxal reductase as a target for the improvement of yeasts' fermentation in industry. Genome Biol Evol. 2019;11:1923-38.

38. Conant GC. Comparative genomics as a time machine: how relative gene dosage and metabolic requirements shaped the time-dependent resolution of yeast polyploidy. Mol Biol Evol. 2014;31:3184-93.

39. Wieczorke R, Krampe S, Weierstall T, Freidel K, Hollenberg CP, Boles E. Concurrent knock-out of at least 20 transporter genes is required to block uptake of hexoses in Saccharomyces cerevisiae. FEBS Lett. 1999;464:123-8.

40. Tripodi F, Nicastro R, Reghellin V, Coccetti P. Post-translational modifications on yeast carbon metabolism: regulatory mechanisms beyond transcriptional control. Biochim Biophys Acta. 2015;1850:620-7.

41. Xu P, Robinson AS. Decreased secretion and unfolded protein response up-regulation are correlated with intracellular retention for single-chain antibody variants produced in yeast. Biotechnol Bioeng. 2009;104:20-9.

42. Yang J, Yan R, Roy A, Xu D, Poisson J, Zhang Y. The I-TASSER Suite: protein structure and function prediction. Nat Methods. 2015;12:7-8.

43. Sun L, Zeng X, Yan C, Sun X, Gong X, Rao Y, Yan N. Crystal structure of a bacterial homologue of glucose transporters GLUT1-4. Nature. 2012;490:361-6.

44. Hoffmann A, Grudinin S. NOLB: nonlinear rigid block normal-mode analysis method. J Chem Theory Comput. 2017;13:2123-34.

45. Tiukova IA, Møller-Hansen I, Belew ZM, Darbani B, Boles E, Nour-Eldin HH, Tomas L, Nielsen J, Boridina I. Identification and characterisation of two high-affinity glucose transporters from the spoilage yeast Brettanomyces bruxellensis. FEMS Microbiol Lett. 2019;366:1-9.

46. Boles $\mathrm{E}$, Hollenberg CP. The molecular genetics of hexose transport in yeasts. FEMS Microbiol Rev. 1997;21:85-111.

47. Snowdon $\mathrm{C}$, van der Merwe G. Regulation of Hxt3 and Hxt7 Turnover Converges on the Vid30 Complex and Requires Inactivation of the Ras/ CAMP/PKA Pathway in Saccharomyces cerevisiae. PLoS ONE. 2012;7:1-10.

48. Brink DP, Borgström C, Tueros FG, Grauslund MFG. Real-time monitoring of the sugar sensing in Saccharomyces cerevisiae indicates endogenous mechanisms for xylose signaling. Microb Cell Fact. 2016;15:183.

49. Osiro KO, Brink DP, Borgström C, Wasserstrom L, Carlquist M, GorwaGrauslund MF. Assessing the effect of D-xylose on the sugar signaling pathways of Saccharomyces cerevisiae in strains engineered for xylose transport and assimilation. FEMS Yeast Res. 2018;18:1-15.

50. Katahira S, Ito M, Takema H, Fujita Y, Tanino T, Tanaka T, Fukuda H, Kondo A. Improvement of ethanol productivity during xylose and glucose cofermentation by xylose-assimilating $S$. cerevisiae via expression of glucose transporter Sut1. Enzyme Microb Technol. 2008:43:115-9.

51. Weierstall T, Hollenberg CP, Boles E. Cloning and characterization of three genes (SUT1-3) encoding glucose transporters of the yeast Pichia stipitis. Mol Microbiol. 1999;31:871-83. 
52. Du J, Li S, Zhao H. Discovery and characterization of novel D-xylose-specific transporters from Neurospora crassa and Pichia stipitis. Mol BioSyst. 2010;6:2150.

53. Moon J, Lewis Liu Z, Ma M, Slininger PJ. New genotypes of industrial yeast Saccharomyces cerevisiae engineered with YXI and heterologous xylose transporters improve xylose utilization and ethanol production. Biocata Agric Biotechnol. 2013;2:247-54.

54. Knoshaug EP, Vidgren V, Magalhães F, Jarvis EE, Franden MA, Zhang $M$, et al. Novel transporters from Kluyveromyces marxianus and Pichia guilliermondii expressed in Saccharomyces cerevisiae enable growth on L-arabinose and D-xylose. Yeast. 2015;32:615-28.

55. Ferreira D, Nobre A, Silva ML, Faria-Oliveira F, Tulha J, Ferreira C, et al. $X Y L H$ encodes a xylose/H+ symporter from the highly related yeast species Debaryomyces fabryi and Debaryomyces hansenii. FEMS Yeast Res. 2013;13:585-96

56. Wang C, Bao X, Li Y, Jiao C, Hou J, Zhang Q, Liu W, Shen Y. Cloning and characterization of heterologous transporters in Saccharomyces cerevisiae and identification of important amino acids for xylose utilization. Metab Eng. 2015;30:79-88.

57. Hector RE, Qureshi N, Hughes SR, Cotta MA. Expression of a heterologous xylose transporter in a Saccharomyces cerevisiae strain engineered to utilize xylose improves aerobic xylose consumption. Appl Microbiol Biotechnol. 2008;80:675-84.

58. Borelli G, José J, Teixeira PJPL, dos Santos LV, Pereira GAG. De novo assembly of Candida sojae and Candida boidinii genomes, unexplored xyloseconsuming yeasts with potential for renewable biochemical production. Genome Announc. 2016:4:e01551-615.

59. Saloheimo A, Rauta J, Stasyk OV, Sibirny AA, Penttilä M, Ruohonen L. Xylose transport studies with xylose-utilizing Saccharomyces cerevisiae strains expressing heterologous and homologous permeases. Appl Microbiol Biotechnol. 2007;74:1041-52.

60. Arruda PV, Santos JC, Rodrigues RCLB, Silva DDV, Yamakawa CK, Rocha GJM, et al. Scale up of xylitol production from sugarcane bagasse hemicellulosic hydrolysate by Candida guilliermondii FTI 20037. J Ind Eng Chem. 2017:47:297-302

61. Mussatto SI, Roberto IC. Xylitol production from high xylose concentration: evaluation of the fermentation in bioreactor under different stirring rates. J Appl Microbiol. 2003:95:331-7.

62. Chen X, Kuhn E, Jennings EW, Nelson R, Tao L, Zhang M, et al. DMR (deacetylation and mechanical refining) processing of corn stover achieves high monomeric sugar concentrations $(230 \mathrm{~g} / \mathrm{L})$ during enzymatic hydrolysis and high ethanol concentrations (>10\% v/v) during fermentation without hydrolysate purification or concentration. Energy Environ Sci. 2016:9:1237-45.

63. Horák J. Regulations of sugar transporters: insights from yeast. Curr Genet. 2013;59:1-31.

64. Krampe S, Boles E. Starvation-induced degradation of yeast hexose transporter Hxt7p is dependent on endocytosis, autophagy and the terminal sequences of the permease. FEBS Lett. 2002:513:193-6.

65. Nijland JG, Vos E, Shin HY, de Waal PP, Klaassen P, Driessen AJM. Improving pentose fermentation by preventing ubiquitination of hexose transporters in Saccharomyces cerevisiae. Biotechnol Biofuels. 2016;9:158.

66. Roy A, Kim Y-B, Cho KH, Kim J-H. Glucose starvation-induced turnover of the yeast glucose transporter Hxt1. Biochim Biophys Acta. 2014; 1840:2878-85.

67. Lane $\mathrm{S}, \mathrm{Xu} \mathrm{H}, \mathrm{Oh}$ EJ, Kim H, Lesmana A, Jeong D, et al. Glucose repression can be alleviated by reducing glucose phosphorylation rate in Saccharomyces cerevisiae. Sci Rep. 2018;8:2613.

68. Subtil T, Boles E. Competition between pentoses and glucose during uptake and catabolism in recombinant Saccharomyces cerevisiae. Biotechnol Biofuels. 2012;5:14.

69. Wisedchaisri G, Park M, ladanza MG, Zheng H, Gonen T. Proton-coupled sugar transport in the prototypical major facilitator superfamily protein XyIE. Nat Commun. 2014;5:4521.

70. Apel AR, Ouellet M, Szmidt-Middleton H, Keasling JD, Mukhopadhyay A. Evolved hexose transporter enhances xylose uptake and glucose/xylose co-utilization in Saccharomyces cerevisiae. Sci Rep. 2016;6:19512.

71. Skjaerven L, Hollup SM, Reuter N. Normal mode analysis for proteins. $J$ Mol Struct THEOCHEM. 2009;898:42-8.

72. Bauer JA, Pavlovíc J, Bauerová-Hlinková V. Normal mode analysis as a routine part of a structural investigation. Molecules. 2019;24:3293.
73. Qureshi AA, Suades A, Matsuoka R, Brock J, Mccomas SE, Nji E, et al. The molecular basis for sugar import in malaria parasites. Nature. 2020:578:321-5.

74. Nijland JG, Shin HY, de Jong RM, de Waal PP, Klaassen P, Driessen AJM. Engineering of an endogenous hexose transporter into a specific D-xylose transporter facilitates glucose-xylose co-consumption in Saccharomyces cerevisiae. Biotechnol Biofuels. 2014;7:168.

75. Ausubel FM, Brent R, Kingston RE, Moore DD, Seidman JG, Smith JA, Struhl, K. Current protocols in molecular biology. Ringbou ed. Wiley; 2003.

76. Cadete RM, Melo MA, Zilli JE, Vital MJS, Mouro A, Prompt AH, et al. Spathaspora brasiliensis sp nov, Spathaspora suhii sp. nov., Spathaspora roraimanensis sp. nov. and Spathaspora xylofermentans sp. nov., four novel D-xylose-fermenting yeast species from Brazilian Amazonian forest. Antonie van Leeuwenhoek. Int J Gen Mol Microbiol. 2013;103:421-31.

77. Wach A, Brachat A, Pöhlmann R, Philippsen P. New heterologous modules for classical or PCR-based gene disruptions in Saccharomyces cerevisiae. Yeast. 1994;10:1793-808.

78. Goldstein AL, McCusker JH. Three new dominant drug resistance cassettes for gene disruption in Saccharomyces cerevisiae. Yeast. 1999:15:1541-53.

79. Gueldener U, Heinisch J, Koehler GJ, Voss D, Hegemann JH. A second set of loxP marker cassettes for Cre-mediated multiple gene knockouts in budding yeast. Nucleic Acids Res. 2002;30:e23.

80. Fell JW, Boekhout T, Fonseca A, Scorzetti G, Statzell-Tallman A. Biodiversity and systematics of basidiomycetous yeasts as determined by largesubunit rDNA D1/D2 domain sequence analysis. Int J Syst Evol Microbiol. 2000:50:1351-71.

81. Tamura K, Stecher G, Peterson D, Filipski A, Kumar S. MEGA6: molecular evolutionary genetics analysis version 6.0. Mol Biol Evol. 2013;30:2725-9.

82. Kurtzman CP. Use of gene sequence analyses and genome comparisons for yeast systematics. Int J Syst Evol Microbiol. 2014;64:325-32.

83. Li L. OrthoMCL: identification of ortholog groups for eukaryotic genomes. Genome Res. 2003;13:2178-89.

84. Katoh K, Standley DM. MAFFT multiple sequence alignment software version 7: improvements in performance and usability article fast track. Mol Biol Evol. 2013:30:772-80.

85. Gibson DG, Young L, Chuang RY, Venter JC, Hutchison CA, Smith HO. Enzymatic assembly of DNA molecules up to several hundred kilobases. Nat Methods. 2009;6:343-5.

86. Christianson TW, Sikorski RS, Dante M, Shero JH, Hieter P. Multifunctional yeast high-copy-number shuttle vectors. Gene. 1992;110:119-22.

87. Gietz RD, Schiestl RH. Large-scale high-efficiency yeast transformation using the LiAc/SS carrier DNA/PEG method. Nat Protoc. 2007;2:38-41.

88. Kaishima M, Ishii J, Matsuno T, et al. Expression of varied GFPs in Saccharomyces cerevisiae: codon optimization yields stronger than expected expression and fluorescence intensity. Sci Rep. 2016;6:1-15.

89. Lang PT, Brozell SR, Mukherjee S, Pettersen EF, Meng EC, Thomas V, et al. DOCK 6: combining techniques to model RNA-small molecule complexes. RNA. 2009:15:1219-30.

90. Pettersen EF, Goddard TD, Huang CC, Couch GS, Greenblatt DM, Meng EC, et al. UCSF Chimera-a visualization system for exploratory research and analysis. J Comput Chem. 2004;25:1605-12.

91. Hess B, Kutzner C, van der Spoel D, Lindahl E. GROMACS 4: algorithms for highly efficient, load-balanced, and scalable molecular simulation. J Chem Theory Comput. 2008:4:435-47.

92. Paulsen PA, Custódio TF, Pedersen BP. Crystal structure of the plant symporter STP10 illuminates sugar uptake mechanism in monosaccharide transporter superfamily. Nat Commun. 2019;10:407.

93. Iancu CV, Zamoon J, Woo SB, Aleshin A, Choe J. Crystal structure of a glucose/H+ symporter and its mechanism of action. Proc Natl Acad Sci USA. 2014;110:17862-7

94. Sikorski RS, Hieter P. A system of shuttle vectors and yeast host strains designed for efficient manipulation of DNA in Saccharomyces cerevisiae. Genetics. 1989:122:19-27.

\section{Publisher's Note}

Springer Nature remains neutral with regard to jurisdictional claims in published maps and institutional affiliations. 